MPI-PhT/95-88

TUM-T31-97/95

hep-ph/9509329

September 1995

\title{
Theoretical Review of B-Physics ${ }^{t}$
}

\author{
AndRzej J. Buras \\ Technische Universität München, Physik Department \\ D-85748 Garching, Germany \\ Max-Planck-Institut für Physik \\ - Werner-Heisenberg-Institut - \\ Föhringer Ring 6, D-80805 München, Germany
}

\begin{abstract}
We review several aspects of B-Physics. In particular we discuss: i) The theoretical framework for B-decays, ii) Weak decays beyond leading logarithms, iii) Standard analysis of the unitarity triangle, iv) Rare B-decays, v) CP violation including the issue of electroweak penguins and vi) Future visions of this field.
\end{abstract}

\footnotetext{
${ }^{1}$ Based on two invited talks given at "Beauty 95", Oxford, July 9-14, 1995, to appear in the proceedings.

Supported by the German Bundesministerium für Bildung und Forschung under contract 06 TM 743 and by the CEC science project SC1-CT91-0729.

e-mail: buras @ feynman.t30.physik.tu-muenchen.de
} 


\title{
Theoretical Review of B-Physics
}

\author{
ANDRZEJ J. Buras \\ Technische Universität München, Physik Department \\ D-85748 Garching, Germany \\ Max-Planck-Institut für Physik \\ - Werner-Heisenberg-Institut - \\ Föhringer Ring 6, D-80805 München, Germany
}

\begin{abstract}
We review several aspects of B-Physics. In particular we discuss: i) The theoretical framework for B-decays, ii) Weak decays beyond leading logarithms, iii) Standard analysis of the unitarity triangle, iv) Rare B-decays, v) CP violation including the issue of electroweak penguins and vi) Future visions of this field.
\end{abstract}

\section{Introduction}

The theoretical review of B-physics presented below is based on two talks I have given at "Beauty 95" held in Oxford in July this year. The first talk dealt with the existing theoretical framework for B-decays. The second talk, the final talk of the workshop, was a theoretical review of B-physics.

It is not my intention to discuss all aspects of B-physics here. I would rather like to concentrate on certain topics in B-physics which I find to be particularly interesting and which I expect to play an important role during this and the following decade. Undoubtly the choice of these topics is dictated to a large extent by my own research in this field, but afterall this is the part of B-physics which I know best and consequently there is some probability that certain messages given below will turn out to be useful. I have organized the material as follows:

Section 2 deals with the theoretical framework for B-decays which is based on the operator product expansion (OPE), the renormalization group, Heavy Quark Effective Theory (HQET) and Heavy Quark Expansions (HQE). Only main ideas are presented there and the technical details are left out. A brief description of the "penguin-box expansion" (PBE) is also given. The latter can be regarded as a version of the OPE which is particularly useful for the study of the $m_{t}$ dependence in weak decays. Finally the status of higher order QCD corrections in weak decays is briefly summarized.

Section 3 discusses the by now standard analysis of the unitarity triangle (UT). After recalling the Wolfenstein parametrization and several basic formulae, I will collect a few messages which should be useful for the UT practitioners. Next the input parameters: 
$\left|V_{c b}\right|,\left|V_{u b} / V_{c b}\right|, B_{K}, F_{B} \sqrt{B_{B}}$ and $m_{t}$ will be discussed. Finally results for the UT and related quantities of interest will be presented.

Section 4 deals with three topics: the semi-leptonic branching ratio $\left(B_{S L}\right)$, the number of charmed hadrons per B-decay $\left(n_{c}\right)$ and the question of factorization in nonleptonic B-decays.

Section 5 summarizes the present status of the three stars in the field of rare B decays: $B \rightarrow X_{s} \gamma, B \rightarrow X_{s} e^{+} e^{-}$and $B \rightarrow \mu \bar{\mu}$.

Section 6 can be regarded as an express review of $\mathrm{CP}$ violation in B-decays. After a list of decays which allow theoretically clean determinations of CKM phases, I will in particular discuss the hot subject of this year: the issue of electroweak penguins in B-decays.

Section 7 is an attempt to classify B- and K-decays from the point of view of theoretical cleanliness and their importance.

Whereas section 8 presents some future visions of the field of weak decays, section 9 is a brief summary. Finally section 10 contains some remarks on "Beauty 95".

\section{Theoretical Framework}

\subsection{Effective Field Theory Picture}

The basic framework for weak decays of hadrons containing $\mathrm{u}, \mathrm{d}, \mathrm{s}, \mathrm{c}$ and $\mathrm{b}$ quarks is the effective field theory relevant for scales $\mu \ll M_{W}, M_{Z}, m_{t}$. This framework brings in local operators which govern "effectively" the transitions in question. From the point of view of the decaying hadrons containing the lightest five quarks this is the only correct picture we know and also the most efficient one for studying the presence of QCD. Furthermore it represents the generalization of the Fermi theory as formulated by Feynman and Gell-Mann [1] almost forty years ago.

Indeed the simplest effective hamiltonian without QCD effects which one would find from the first diagram of fig. 1 is

$$
H_{e f f}^{o}=\frac{G_{F}}{\sqrt{2}} V_{c b} V_{c s}^{*}(\bar{c} b)_{V-A}(\bar{s} c)_{V-A}
$$

where $G_{F}$ is the Fermi constant, $V_{i j}$ are the relevant CKM factors and

$$
(\bar{c} b)_{V-A}(\bar{s} c)_{V-A} \equiv\left(\bar{c} \gamma_{\mu}\left(1-\gamma_{5}\right) b\right)\left(\bar{s} \gamma_{\mu}\left(1-\gamma_{5}\right) c\right)=Q_{2}
$$

is a $(V-A) \cdot(V-A)$ current-current local operator denoted usually by $Q_{2}$. The situation in the standard model is however more complicated because of the presence of additional interactions which effectively generate new operators. These are in particular the gluon, photon and $Z^{0}$-boson exchanges and internal top contributions. Some of the elementary interactions of this type are shown in fig. 1. Consequently the relevant effective hamiltonian for B-meson decays involves generally several operators $Q_{i}$ with 
various colour and Dirac structures which are different from $Q_{2}$. Moreover each operator is multiplied by a calculable coefficient $C_{i}(\mu)$ :

$$
H_{e f f}=\frac{G_{F}}{\sqrt{2}} V_{C K M} \sum_{i} C_{i}(\mu) Q_{i}
$$

In this connection it should be mentioned that the usual Feynman diagram drawings of the type shown in fig. 1 containing full $\mathrm{W}$-propagators, $Z^{0}$-propagators and top-quark propagators represent really the happening at scales $\mathcal{O}\left(M_{W}\right)$ whereas the true picture of a decaying hadron is more correctly described by effective vertices which are represented by the local operators in question. Thus, whereas at scales $\mathcal{O}\left(M_{W}\right)$ we deal with the full six-quark theory containing the photon, weak gauge bosons and gluons, at scales $\mathcal{O}(1 \mathrm{GeV})$ the relevant effective theory contains only three light quarks $\mathrm{u}$, $\mathrm{d}$ and $\mathrm{s}$, gluons and the photon. At intermediate energy scales, $\mu=\mathcal{O}\left(m_{b}\right)$ and $\mu=\mathcal{O}\left(m_{c}\right)$, relevant for beauty and charm decays effective five-quark and effective four-quark theories have to be considered respectively.

The usual procedure then is to start at a high energy scale $\mathcal{O}\left(M_{W}\right)$ and consecutively integrate out the heavy degrees of freedom (heavy with respect to the relevant scale $\mu$ ) from explicitly appearing in the theory. The word "explicitly" is very essential here. The heavy fields did not disappear. Their effects are merely hidden in the effective gauge coupling constants, running masses and most importantly in the coefficients describing the "effective" strength of the operators at a given scale $\mu$, the Wilson coefficient functions $C_{i}(\mu)$.

\subsection{OPE and Renormalization Group}

The Operator Product Expansion (OPE) combined with the renormalization group approach can be regarded as a mathematical formulation of the picture outlined above. In this framework the amplitude for an exclusive decay $M \rightarrow F$ is written as

$$
A(M \rightarrow F)=\frac{G_{F}}{\sqrt{2}} V_{C K M} \sum_{i} C_{i}(\mu)\left\langle F\left|Q_{i}(\mu)\right| M\right\rangle
$$

where $M$ stands for the decaying meson, $F$ for a given final state and $V_{C K M}$ denotes the relevant $C K M$ factor. $Q_{i}(\mu)$ denote the local operators generated by QCD and electroweak interactions. $C_{i}(\mu)$ stand for the Wilson coefficient functions (c-numbers). The scale $\mu$ separates the physics contributions in the "short distance" contributions (corresponding to scales higher than $\mu$ ) contained in $C_{i}(\mu)$ and the "long distance" contributions (scales lower than $\mu$ ) contained in $\left\langle F\left|Q_{i}(\mu)\right| M\right\rangle$. By evolving the scale from $\mu=\mathcal{O}\left(M_{W}\right)$ down to lower values of $\mu$ one transforms the physics information at scales higher than $\mu$ from the hadronic matrix elements into $C_{i}(\mu)$. Since no information is lost this way the full amplitude cannot depend on $\mu$. This is the essence of the renormalization group equations which govern the evolution $\left(\mu-\right.$ dependence) of $C_{i}(\mu)$. This $\mu$-dependence must be cancelled by the one present in $\left\langle Q_{i}(\mu)\right\rangle$. It should be 
stressed, however, that this cancellation generally involves many operators due to the operator mixing under renormalization.

The general expression for $C_{i}(\mu)$ is given by:

$$
\vec{C}(\mu)=\hat{U}\left(\mu, M_{W}\right) \vec{C}\left(M_{W}\right)
$$

where $\vec{C}$ is a column vector built out of $C_{i}$ 's. $\vec{C}\left(M_{W}\right)$ are the initial conditions which depend on the short distance physics at high energy scales. In particular they depend on $m_{t} . \hat{U}\left(\mu, M_{W}\right)$, the evolution matrix, is given as follows:

$$
\hat{U}\left(\mu, M_{W}\right)=T_{g} \exp \left[\int_{g\left(M_{W}\right)}^{g(\mu)} d g^{\prime} \frac{\hat{\gamma}^{T}\left(g^{\prime}\right)}{\beta\left(g^{\prime}\right)}\right]
$$

with $g$ denoting the QCD effective coupling constant. $\beta(g)$ governs the evolution of $g$ and $\hat{\gamma}$ is the anomalous dimension matrix of the operators involved. The structure of this equation makes it clear that the renormalization group approach goes beyond the usual perturbation theory. Indeed $\hat{U}\left(\mu, M_{W}\right)$ sums automatically large logarithms $\log M_{W} / \mu$ which appear for $\mu<<M_{W}$. In the so-called leading logarithmic approximation (LO) terms $\left(g^{2} \log M_{W} / \mu\right)^{n}$ are summed. The next-to-leading logarithmic correction (NLO) to this result involves summation of terms $\left(g^{2}\right)^{n}\left(\log M_{W} / \mu\right)^{n-1}$ and so on. This hierarchic structure gives the renormalization group improved perturbation theory.

As an example let us consider only QCD effects and the case of a single operator so that (5) reduces to

$$
C(\mu)=U\left(\mu, M_{W}\right) C\left(M_{W}\right)
$$

with $C(\mu)$ denoting the coefficient of the operator in question. Keeping the first two terms in the expansions of $\gamma(g)$ and $\beta(g)$ in powers of $g$ :

$$
\gamma(g)=\gamma^{(0)} \frac{\alpha_{s}}{4 \pi}+\gamma^{(1)} \frac{\alpha_{s}^{2}}{16 \pi^{2}} \quad, \quad \beta(g)=-\beta_{0} \frac{g^{3}}{16 \pi^{2}}-\beta_{1} \frac{g^{5}}{\left(16 \pi^{2}\right)^{2}}
$$

and inserting these expansions into (6) gives:

$$
U\left(\mu, M_{W}\right)=\left[1+\frac{\alpha_{s}(\mu)}{4 \pi} J\right]\left[\frac{\alpha_{s}\left(M_{W}\right)}{\alpha_{s}(\mu)}\right]^{P}\left[1-\frac{\alpha_{s}\left(M_{W}\right)}{4 \pi} J\right]
$$

where

$$
P=\frac{\gamma^{(0)}}{2 \beta_{0}}, \quad J=\frac{P}{\beta_{0}} \beta_{1}-\frac{\gamma^{(1)}}{2 \beta_{0}} .
$$

General formulae for $\hat{U}\left(\mu, M_{W}\right)$ in the case of operator mixing and valid also for electroweak effects can be found in [2]. The leading logarithmic approximation corresponds to setting $J=0$ in (9).

Clearly in order to calculate the full amplitude in (困) also the matrix elements $\left\langle F\left|Q_{i}(\mu)\right| M\right\rangle$ have to be evaluated. Since they involve long distance contributions 
one is forced in this case to use non-perturbative methods such as lattice calculations, the $1 / N$ expansion, QCD sum rules or chiral perturbation theory. In the case of semileptonic B meson decays also the Heavy Quark Effective Theory (HQET) [3] turns out to be a useful tool. In HQET the matrix elements are evaluated approximately in an expansion in $1 / m_{b}$. Needless to say all these non-perturbative methods have some limitations. Consequently the dominant theoretical uncertainties in the decay amplitudes reside in the matrix elements of $Q_{i}$.

Fig. 1 


\subsection{Classification of Operators}

Below we give six classes of operators which play the dominant role in the phenomenology of weak decays. Typical diagrams in the full theory from which these operators originate are indicated and shown in Fig. 1. The cross in Fig. 1d indicates that magnetic penguins originate from the mass-term on the external line in the usual QCD or QED penguin diagrams. The six classes are given as follows ( $\alpha$ and $\beta$ are colour indices):

Current-Current (Fig. 1a):

$$
Q_{1}=\left(\bar{c}_{\alpha} b_{\beta}\right)_{V-A}\left(\bar{s}_{\beta} c_{\alpha}\right)_{V-A} \quad Q_{2}=(\bar{c} b)_{V-A}(\bar{s} c)_{V-A}
$$

QCD-Penguins (Fig. 1b):

$$
\begin{aligned}
Q_{3} & =(\bar{s} b)_{V-A} \sum_{q=u, d, s, c}(\bar{q} q)_{V-A} & Q_{4} & =\left(\bar{s}_{\alpha} b_{\beta}\right)_{V-A} \sum_{q=u, d, s, c}\left(\bar{q}_{\beta} q_{\alpha}\right)_{V-A} \\
Q_{5} & =(\bar{s} b)_{V-A} \sum_{q=u, d, s, c}(\bar{q} q)_{V+A} & Q_{6} & =\left(\bar{s}_{\alpha} b_{\beta}\right)_{V-A} \sum_{q=u, d, s, c}\left(\bar{q}_{\beta} q_{\alpha}\right)_{V+A}
\end{aligned}
$$

Electroweak-Penguins (Fig. 1c):

$$
\begin{aligned}
Q_{7} & =\frac{3}{2}(\bar{s} b)_{V-A} \sum_{q=u, d, s, c} e_{q}(\bar{q} q)_{V+A} & Q_{8} & =\frac{3}{2}\left(\bar{s}_{\alpha} b_{\beta}\right)_{V-A} \sum_{q=u, d, s, c} e_{q}\left(\bar{q}_{\beta} q_{\alpha}\right)_{V+A} \\
Q_{9} & =\frac{3}{2}(\bar{s} b)_{V-A} \sum_{q=u, d, s, c} e_{q}(\bar{q} q)_{V-A} & Q_{10} & =\frac{3}{2}\left(\bar{s}_{\alpha} b_{\beta}\right)_{V-A} \sum_{q=u, d, s, c} e_{q}\left(\bar{q}_{\beta} q_{\alpha}\right)_{V-A}
\end{aligned}
$$

Magnetic-Penguins (Fig. 1d):

$$
Q_{7 \gamma}=\frac{e}{8 \pi^{2}} m_{b} \bar{s}_{\alpha} \sigma^{\mu \nu}\left(1+\gamma_{5}\right) b_{\alpha} F_{\mu \nu} \quad Q_{8 G}=\frac{g}{8 \pi^{2}} m_{b} \bar{s}_{\alpha} \sigma^{\mu \nu}\left(1+\gamma_{5}\right) T_{\alpha \beta}^{a} b_{\beta} G_{\mu \nu}^{a}
$$

$\Delta S=2$ and $\Delta B=2$ Operators (Fig. 3e):

$$
Q(\Delta S=2)=(\bar{s} d)_{V-A}(\bar{s} d)_{V-A} \quad Q(\Delta B=2)=(\bar{b} d)_{V-A}(\bar{b} d)_{V-A}
$$

Semi-Leptonic Operators (Fig. 1f):

$$
Q_{9 V}=(\bar{s} b)_{V-A}(\bar{e} e)_{V} \quad Q_{10 A}=(\bar{s} b)_{V-A}(\bar{e} e)_{A}
$$

\subsection{Towards Phenomenology}

The rather formal expression for the decay amplitudes given in (4) can always be cast in the form 画:

$$
A(M \rightarrow F)=\sum_{i} B_{i} V_{C K M}^{i} \eta_{Q C D}^{i} F_{i}\left(m_{t}, m_{c}\right)
$$

which is more useful for phenomenology. In writing (19) we have generalized (4) to include several CKM factors. $F_{i}\left(m_{t}, m_{c}\right)$, the Inami-Lim functions, result from the evaluation of loop diagrams with internal top and charm exchanges (see fig. 1) and may 
also depend solely on $m_{t}$ or $m_{c}$. In the case of current-current operators the $F_{i}$ are mass independent. The factors $\eta_{Q C D}^{i}$ summarize the QCD corrections which can be calculated by formal methods discussed above. Finally $B_{i}$ stand for nonperturbative factors related to the hadronic matrix elements of the contributing operators: the main theoretical uncertainty in the whole enterprise. In leptonic and semi-leptonic decays for which only the matrix elements of weak currents are needed, the non-perturbative $B$-factors can fortunately be determined from leading tree level decays reducing or removing the nonperurbative uncertainty. In non-leptonic decays this is generally not possible and we have to rely on existing non-perturbative methods. A well known example of a $B_{i}$-factor is the renormalization group invariant parameter $B_{K}$ [5] defined by

$$
B_{K}=B_{K}(\mu)\left[\alpha_{s}(\mu)\right]^{-2 / 9} \quad\left\langle\bar{K}^{o}|Q(\Delta S=2)| K^{o}\right\rangle=\frac{8}{3} B_{K}(\mu) F_{K}^{2} m_{K}^{2}
$$

In order to simplify the presentation we have omitted the NLO correction in $B_{K} \cdot B_{K}$ plays an important role in the phenomenology of $\mathrm{CP}$ violation in $K \rightarrow \pi \pi$.

If one is interested in exhibiting the $m_{t}$ dependence, the expression (19) can further be rewritten [4] as a linear combination of seven basic, universal (process independent) $m_{t^{-}}$ dependent functions $F_{r}\left(x_{t}\right)$ with coefficients $P_{r}$ characteristic for the decay considered:

$$
A(M \rightarrow F)=P_{o}+\sum_{r} P_{r} F_{r}\left(x_{t}\right) \quad, \quad x_{t}=\frac{m_{t}^{2}}{M_{W}^{2}}
$$

where the sum is over all possible functions contributing to a given amplitude. The first term is related to contributions involving other quarks, in particular the charm quark. This is the Penguin-Box Expansion (PBE) mentoned previously.

Equation (21) can be derived from the usual OPE by setting $\mu=M_{W}$ and decomposing properly the $m_{t}$ dependence of different $C_{i}\left(M_{W}\right)$ into the basic functions $F_{r}\left(x_{t}\right)$ which result from the diagrams of fig. 1. The latter have been evaluated by Inami and Lim [6]. Since the $\gamma$-penguins, $Z^{0}$-penguins and $\Delta S=1$ box diagrams are gauge dependent, the idea of 44 was to consider a set of functions $F_{r}\left(x_{t}\right)$ corresponding to gauge independent combinations. The approximate expressions for $F_{r}\left(x_{t}\right)$ are given as follows:

$$
\begin{gathered}
S\left(x_{t}\right)=0.784 x_{t}^{0.76}, \quad X\left(x_{t}\right)=0.660 x_{t}^{0.575}, \\
Y\left(x_{t}\right)=0.315 x_{t}^{0.78}, \quad Z\left(x_{t}\right)=0.175 x_{t}^{0.93}, \quad E\left(x_{t}\right)=0.564 x_{t}^{-0.51}, \\
D^{\prime}\left(x_{t}\right)=0.244 x_{t}^{0.30}, \quad E^{\prime}\left(x_{t}\right)=0.145 x_{t}^{0.19} .
\end{gathered}
$$

In the range $150 \mathrm{GeV} \leq m_{t} \leq 200 \mathrm{GeV}$ these approximations reproduce the exact expressions to an accuracy beter than $1 \%$. $S\left(x_{t}\right)$ results from the $\Delta S=2$ or $\Delta B=2$ box diagram, $E\left(x_{t}\right)$ from the gluon penguin, $D^{\prime}\left(x_{t}\right)$ from the magnetic $\gamma$-penguin and $E^{\prime}\left(x_{t}\right)$ from the magnetic gluon penguin. $X\left(x_{t}\right)$ and $Y\left(x_{t}\right)$ are linear combinations of the $\mathrm{V}$-A component of $Z^{0}$-penguin and box-diagrams with final quarks or leptons having $T_{3}$ (weak isospin) equal to $1 / 2$ and $-1 / 2$ respectively. Finally $Z\left(x_{t}\right)$ is a linear combination of the vector component of the $Z^{0}$-penguin and the $\gamma$-penguin. 


\begin{tabular}{|c|c|}
\hline Decay & Reference \\
\hline \multicolumn{2}{|l|}{$\Delta F=1$ Decays } \\
\hline current-current operators & [13, 14] \\
\hline QCD penguin operators & [16, 2, 18, 19 \\
\hline electroweak penguin operators & 《17, 2, 18, 19 \\
\hline magnetic penguin operators & 20] \\
\hline$B r(B)_{S L}$ & [13, 21, 22] \\
\hline inclusive $\Delta S=1$ decays & 23 \\
\hline \multicolumn{2}{|c|}{$\begin{array}{l}\text { Particle-Antiparticle Mixing } \\
\end{array}$} \\
\hline$\eta_{1}$ & 24 \\
\hline$\eta_{2}, \eta_{B}$ & 25 \\
\hline$\eta_{3}$ & 26 \\
\hline \multicolumn{2}{|c|}{ Rare K- and B-Meson Decays } \\
\hline$K_{L}^{0} \rightarrow \pi^{0} \nu \bar{\nu}, B \rightarrow l^{+} l^{-}, B \rightarrow X_{\mathrm{s}} \nu \bar{\nu}$ & 27,28 \\
\hline$K^{+} \rightarrow \pi^{+} \nu \bar{\nu}, K_{L} \rightarrow \mu^{+} \mu^{-}$ & 29 \\
\hline$K^{+} \rightarrow \pi^{+} \mu \bar{\mu}$ & 30 \\
\hline$K_{L} \rightarrow \pi^{0} e^{+} e^{-}$ & 31 \\
\hline$B \rightarrow X_{s} e^{+} e^{-}$ & 32,33 \\
\hline
\end{tabular}

Table 1: References to NLO Calculations

The coefficients $P_{r}$ include the physics from scales $0 \leq \mu \leq M_{W}$ and also the CKM factors which have been shown explicitly in (19). Generally in a given decay several of the coefficients vanish so that the corresponding amplitude depends only on one or a few functions.

\subsection{Inclusive Decays}

So far we have discussed only exclusive decays. During the recent years considerable progress has been made for inclusive decays of heavy mesons. The starting point is again the effective hamiltonian in (3) which includes the short distance QCD effects in $C_{i}(\mu)$. The actual decay described by the operators $Q_{i}$ is then calculated in the spectator model corrected for additional virtual and real gluon corrections. Support for this approximation comes from heavy quark $\left(1 / m_{b}\right)$ expansions (HQE). Indeed the spectator model has been shown to correspond to the leading order approximation in the $1 / m_{b}$ expansion. The next corrections appear at the $\mathcal{O}\left(1 / m_{b}^{2}\right)$ level. The latter terms have been studied by several authors [0, 8, 9] with the result that they affect various branching ratios by less than $10 \%$ and often by only a few percent. There is a vast literature on this subject and I can only refer here to a few papers [9, 10] where further references can be found. Of particular importance for this field was also the issue of the 
renormalons which are nicely discussed in [11, 12].

\subsection{Weak Decays Beyond Leading Logarithms}

Until 1989 most of the calculations in the field of weak decays were done in the leading logarithmic approximation. An exception was the important work of Altarelli et al. [13] who in 1981 calculated NLO QCD corrections to the Wilson coefficients of the current-current operators.

Today the effective hamiltonians for weak decays are available at the next-to-leading level for the most important and interesting cases due to a series of publications devoted to this enterprise beginning with the work of Peter Weisz and myself in 1989 [14]. The list of the existing calculations is given in table 1. We will discuss some of the entries in this list below. A detailed review of the existing NLO calculations will appear soon [15.].

Let us recall why NLO calculations are important for the phenomenology of weak decays:

- The NLO is first of all necessary to test the validity of the renormalization group improved perturbation theory.

- Without going to NLO the QCD scale $\Lambda_{\overline{M S}}$ extracted from various high energy processes cannot be used meaningfully in weak decays.

- Due to the renormalization group invariance the physical amplitudes do not depend on the scales $\mu$ present in $\alpha_{s}$ or in the running quark masses, in particular $m_{t}(\mu), m_{b}(\mu)$ and $m_{c}(\mu)$. However in perturbation theory this property is broken through the truncation of the perturbative series. Consequently one finds sizable scale ambiguities in the leading order, which can be reduced considerably by going to NLO.

- In several cases the central issue of the top quark mass dependence is strictly a NLO effect.

\section{Standard Analysis}

\subsection{The CKM Matrix and the Unitarity Triangle}

An important target of particle physics is the determination of the unitary $3 \times 3$ CabibboKobayashi-Maskawa matrix [34, 35] which parametrizes the charged current interactions of quarks:

$$
J_{\mu}^{c c}=(\bar{u}, \bar{c}, \bar{t})_{L} \gamma_{\mu}\left(\begin{array}{ccc}
V_{u d} & V_{u s} & V_{u b} \\
V_{c d} & V_{c s} & V_{c b} \\
V_{t d} & V_{t s} & V_{t b}
\end{array}\right)\left(\begin{array}{c}
d \\
s \\
b
\end{array}\right)_{L}
$$


The CP violation in the standard model is supposed to arise from a single phase in this matrix. It is customary these days to express the CKM-matrix in terms of four Wolfenstein parameters [36] $(\lambda, A, \varrho, \eta)$ with $\lambda=\left|V_{u s}\right|=0.22$ playing the role of an expansion parameter and $\eta$ representing the $\mathrm{CP}$ violating phase:

$$
V_{C K M}=\left(\begin{array}{ccc}
1-\frac{\lambda^{2}}{2} & \lambda & A \lambda^{3}(\varrho-i \eta) \\
-\lambda & 1-\frac{\lambda^{2}}{2} & A \lambda^{2} \\
A \lambda^{3}(1-\varrho-i \eta) & -A \lambda^{2} & 1
\end{array}\right)+O\left(\lambda^{4}\right)
$$

Because of the smallness of $\lambda$ and the fact that for each element the expansion parameter is actually $\lambda^{2}$, it is sufficient to keep only the first few terms in this expansion.

Fig. 2

Following [37] one can define the parameters $(\lambda, A, \varrho, \eta)$ through

$$
s_{12} \equiv \lambda \quad s_{23} \equiv A \lambda^{2} \quad s_{13} e^{-i \delta} \equiv A \lambda^{3}(\varrho-i \eta)
$$

where $s_{i j}$ and $\delta$ enter the standard exact parametrization 38] of the CKM matrix. This specifies the higher orders terms in (26).

The definition of $(\lambda, A, \varrho, \eta)$ given in (27) is useful because it allows to improve the accuracy of the original Wolfenstein parametrization in an elegant manner. In particular

$$
\begin{array}{cc}
V_{u s}=\lambda & V_{c b}=A \lambda^{2} \\
V_{u b}=A \lambda^{3}(\varrho-i \eta) & V_{t d}=A \lambda^{3}(1-\bar{\varrho}-i \bar{\eta})
\end{array}
$$

where

$$
\bar{\varrho}=\varrho\left(1-\frac{\lambda^{2}}{2}\right) \quad \bar{\eta}=\eta\left(1-\frac{\lambda^{2}}{2}\right)
$$

turn out [37 to be excellent approximations to the exact expressions.

A useful geometrical representation of the CKM matrix is the unitarity triangle obtained by using the unitarity relation

$$
V_{u d} V_{u b}^{*}+V_{c d} V_{c b}^{*}+V_{t d} V_{t b}^{*}=0
$$


rescaling it by $\left|V_{c d} V_{c b}^{*}\right|=A \lambda^{3}$ and depicting the result in the complex $(\bar{\rho}, \bar{\eta})$ plane as shown in fig. 2. The lenghts $\mathrm{CB}, \mathrm{CA}$ and $\mathrm{BA}$ are equal respectively to 1 ,

$$
R_{b} \equiv \sqrt{\bar{\varrho}^{2}+\bar{\eta}^{2}}=\left(1-\frac{\lambda^{2}}{2}\right) \frac{1}{\lambda}\left|\frac{V_{u b}}{V_{c b}}\right| \quad \text { and } \quad R_{t} \equiv \sqrt{(1-\bar{\varrho})^{2}+\bar{\eta}^{2}}=\frac{1}{\lambda}\left|\frac{V_{t d}}{V_{c b}}\right| .
$$

The triangle in fig. 2, $\left|V_{u s}\right|$ and $\left|V_{c b}\right|$ give the full description of the CKM matrix. Looking at the expressions for $R_{b}$ and $R_{t}$ we observe that within the standard model the measurements of four CP conserving decays sensitive to $\left|V_{u s}\right|,\left|V_{u b}\right|,\left|V_{c b}\right|$ and $\left|V_{t d}\right|$ can tell us whether $\mathrm{CP}$ violation $(\eta \neq 0)$ is predicted in the standard model. This is a very remarkable property of the Kobayashi-Maskawa picture of CP violation: quark mixing and $\mathrm{CP}$ violation are closely related to each other.

There is of course the very important question whether the KM picture of CP violation is correct and more generally whether the standard model offers a correct description of weak decays of hadrons. In order to answer these important questions it is essential to calculate as many branching ratios as possible, measure them experimentally and check if they all can be described by the same set of the parameters $(\lambda, A, \varrho, \eta)$. In the language of the unitarity triangle this means that the various curves in the $(\bar{\varrho}, \bar{\eta})$ plane extracted from different decays should cross each other at a single point. Moreover the angles $(\alpha, \beta, \gamma)$ in the resulting triangle should agree with those extracted one day from $\mathrm{CP}$-asymmetries in B-decays. More about this below.

\subsection{Basic Formulae}

At present there is still a rather limited knowledge of the shape of the unitarity triangle. The standard analysis using the available experimental and theoretical information proceeds essentially in four steps:

\section{Step 1:}

From $b \rightarrow c$ transition in inclusive and exclusive B meson decays one finds $\left|V_{c b}\right|$ and consequently the scale of UT:

$$
\left|V_{c b}\right| \quad=>\lambda\left|V_{c b}\right|=\lambda^{3} A
$$

\section{Step 2:}

From $b \rightarrow u$ transition in inclusive B meson decays one finds $\left|V_{u b} / V_{c b}\right|$ and consequently the side $C A=R_{b}$ of UT:

$$
\left|V_{u b} / V_{c b}\right|=>\quad R_{b}
$$

\section{Step 3:}

From the observed indirect $\mathrm{CP}$ violation in $K \rightarrow \pi \pi$ described experimentally by the parameter $\varepsilon_{K}$ and theoretically by the imaginary part of the relevant box diagram in fig. 1e one derives the constraint:

$$
\bar{\eta}\left[(1-\bar{\varrho}) A^{2} \eta_{2} S\left(x_{t}\right)+P_{0}(\varepsilon)\right] A^{2} B_{K}=0.226
$$


where

$$
P_{0}(\varepsilon)=\left[\eta_{3} S\left(x_{c}, x_{t}\right)-\eta_{1} x_{c}\right] \frac{1}{\lambda^{4}}
$$

Equation (35) specifies a hyperbola in the $(\bar{\varrho}, \bar{\eta})$ plane. Here $S\left(x_{t}\right)$ is the function given in (22), $B_{K}$ is the non-perturbative parameter defined in (20) and $\eta_{2}$ is the QCD factor in the box diagrams with two top quark exchanges. Finally $P_{0}(\varepsilon)=0.31 \pm 0.02$ summarizes the contributions of box diagrams with two charm quark exchanges and the mixed charm-top exchanges. $P_{0}(\varepsilon)$ depends very weakly on $m_{t}$ and its range given above corresponds to $155 \mathrm{GeV} \leq m_{t} \leq 185 \mathrm{GeV}$. The QCD factors $\eta_{1}$ for the (cc) contribution and $\eta_{3}$ for the (ct) contribution are included in this calculation as seen in (36).

\section{Step 4:}

From the observed $B_{d}^{0}-\bar{B}_{d}^{0}$ mixing described experimentally by the mixing parameter $x_{d}=\Delta M / \Gamma_{B}$ and theoretically by the relevant box diagram of Fig. 1e the side $B A=R_{t}$ of the UT can be determined:

$$
R_{t}=1.52 \cdot \frac{R_{0}}{\sqrt{S\left(x_{t}\right)}}=0.97 R_{0} \cdot\left[\frac{170 G e V}{\bar{m}_{t}\left(m_{t}\right)}\right]^{0.76}
$$

where

$$
R_{0} \equiv \sqrt{\frac{x_{d}}{0.75}}\left[\frac{200 M e V}{F_{B_{d}} \sqrt{B_{B_{d}}}}\right]\left[\frac{0.040}{\left|V_{c b}\right|}\right]\left[\frac{1.6 p s}{\tau_{B}}\right]^{0.5} \sqrt{\frac{0.55}{\eta_{B}}}
$$

This allows to determine $\left|V_{t d}\right|$ :

$$
\left|V_{t d}\right|=8.6 \cdot 10^{-3}\left[\frac{200 \mathrm{MeV}}{\sqrt{B_{B}} F_{B}}\right]\left[\frac{170 \mathrm{GeV}}{\bar{m}_{t}\left(m_{t}\right)}\right]^{0.76}\left[\frac{x_{d}}{0.75}\right]^{0.5}\left[\frac{1.6 p s}{\tau_{B}}\right]^{0.5} \sqrt{\frac{0.55}{\eta_{B}}}
$$

Here $\tau_{B}$ is the B-meson life-time, $\eta_{B}$ is the QCD factor analogous to $\eta_{2}, F_{B_{d}}$ is the B-meson decay constant and $B_{B_{d}}$ denotes a non-perturbative parameter analogous to $B_{K}$. Note that whereas $R_{t}$ depends on $\left|V_{c b}\right|$, the determination of $\left|V_{t d}\right|$ by means of $x_{d}$ is free from this dependence.

Before discussing the values of the input parameters in the basic formulae given above we would like to make a few important messages for UT-practitioners.

\subsection{Messages for UT Practitioners}

\subsubsection{Message 1}

The parameter $m_{t}$, the top quark mass, used in weak decays is not equal to the one used in the electroweak precision studies at LEP or SLD. In the latter investigations the so-called pole mass is used, whereas in all the NLO calculations listed in table $1 m_{t}$ refers to the running current top quark mass normalized at $\mu=m_{t}: \bar{m}_{t}\left(m_{t}\right)$. One has

$$
m_{t}^{\text {Pole }}=\bar{m}_{t}\left(m_{t}\right)\left[1+\frac{4}{3} \frac{\alpha_{s}\left(m_{t}\right)}{\pi}\right]
$$


so that for $m_{t}=\mathcal{O}(170 \mathrm{GeV}), \bar{m}_{t}\left(m_{t}\right)$ is typically by $8 \mathrm{GeV}$ smaller than $m_{t}^{\text {Pole }}$. This difference will matter in a few years. There is also an interesting question: What is the meaning of $m_{t}$ quoted by CDF [39] and D0 [40]? My feeling is that this is unclear at present. I will assume, as most people do, that this is the pole mass and consequently use in weak decays

$$
m_{t} \equiv \bar{m}_{t}\left(m_{t}\right)=(170 \pm 15) G e V
$$

In principle an error of $11 \mathrm{GeV}$ could be used. In view of the uncertainty in the definition of $m_{t}$ I prefer to be conservative. Let us hope that the issue of the definition of $m_{t}$ at Fermilab will be clarified soon.

\subsubsection{Message 2}

When using numerical values for $m_{t}, B_{K}, B_{B}$ and the QCD factors $\eta_{i}$, care must be taken that they are used consistently. This unfortunately is not always the case. As an example let us consider the theoretical expression for $x_{d}$ which reads

$$
x_{d}=C_{B} F_{B}^{2} B_{B}\left(\mu_{B}\right)\left[\alpha_{s}\left(\mu_{B}\right)\right]^{-6 / 25} \eta_{B}\left(\mu_{t}, \bar{m}_{t}\left(\mu_{t}\right)\right) S\left(\bar{m}_{t}\left(\mu_{t}\right)\right)
$$

with $C_{B}$ being a numerical constant. Here $S$ stands for the box diagram function of (22) and $\eta_{B}$ represents the QCD corrections to the relevant box diagrams. $B_{B}\left(\mu_{B}\right)$ is defined in analogy to $B_{K}(\mu)$ in (20). Two relevant scales are $\mu_{B}=\mathcal{O}\left(m_{b}\right)$ and $\mu_{t}=\mathcal{O}\left(m_{t}\right)$ which according to the rules of the renormalization group game can be chosen for instance in the ranges $2.5 \mathrm{GeV} \leq \mu_{B} \leq 10 \mathrm{GeV}$ and $100 \mathrm{GeV} \leq \mu_{t} \leq 300 \mathrm{GeV}$ respectively. $\mu_{B}$ is the scale at which the relevant $\Delta B=2$ operator is normalized, $\mu_{t}$ is the scale at which $m_{t}$ is defined. Clearly $x_{d}$ cannot depend on $\mu_{B}$ and $\mu_{t}$. Combining the explicit $\alpha_{s}$ factor in (42) with $B_{B}\left(\mu_{B}\right)$ as in (20) and introducing the renormalization group invariant $B_{B}$ removes $\mu_{B}$ from phenomenological expressions like (39). On the other hand the $\mu_{t}$ dependence cancells between the last two terms as demonstrated explicitly in [25]. To this end the NLO calculation for $\eta_{B}$ is essential. Otherwise $x_{d}$ shows a sizable $\mu_{t}$ dependence. It turns out that for a choice $\mu_{t}=m_{t}, \eta_{B}$ and similarly $\eta_{2}$ in (35) are practically independent of $m_{t}$. This is convenient and has been adopted in 25 and in subsequent NLO calculations. Then $\eta_{B}=0.55$ and $\eta_{2}=0.57$ independent of $m_{t}$.

In "old days" the explicit $\alpha_{s}$ factor in (42) has been combined with $\eta_{B}$ to give the corresponding $\mu_{B}$ dependent QCD factor as high as 0.85 . This change is compensated by $B_{B}\left(\mu_{B}\right)<B_{B}$. In view of the fact that all present "non-perturbative researchers" quote $B_{B}$ and $B_{K}$, it is important that this older definition is abandoned.

Similar messages apply to $\eta_{i}$ in the case of $\varepsilon_{K}$.

\subsubsection{Message 3}

It is sometimes stated in the literature that the QCD factors $\eta_{B}$ for $B_{d}^{0}-\bar{B}_{d}^{0}$ and $B_{s}^{0}-\bar{B}_{s}^{0}$ mixings are "expected" to be equal to each other. There is nothing to expect here. They are equal. Indeed, $\eta_{B}$ resulting from short distance QCD calculations is independent of 
whether $B_{d}^{0}-\bar{B}_{d}^{0}$ or $B_{s}^{0}-\bar{B}_{s}^{0}$ is considered. Consequently the ratio $x_{d} / x_{s}$ is independent of $m_{t}$ and short distance QCD corrections. The only difference in these two mixings arises through different CKM factors, life-times and through different hadronic matrix elements of the relevant $\Delta B=2$ operators which corresponds to $F_{B_{s}} \neq F_{B_{d}}$ and $B_{B_{s}} \neq B_{B_{d}}$. This is explicitly summarized in (50).

\subsection{Input Parameters}

Let us next discuss the input parameters needed to perform the standard analysis.

\subsection{1 $\left|V_{c b}\right|$}

During the last two years there has been a considerable progress done by experimentalists 41] and theorists in the extraction of $\left|V_{c b}\right|$ from exclusive and inclusive decays. In particular I would like to mention important papers by Neubert [42], Shifman, Uraltsev and Vainshtein [43, and Ball, Benecke and Braun [12] on the basis of which one is entitled to use ( for $\tau_{B}=1.6 \mathrm{ps}$ ):

$$
\left|V_{c b}\right|=0.040 \pm 0.003 \quad \Rightarrow \quad A=0.82 \pm 0.06
$$

\subsection{2 $\left|V_{u b} / V_{c b}\right|$}

Here the situation is much worse and the value

$$
\left|\frac{V_{u b}}{V_{c b}}\right|=0.08 \pm 0.02
$$

quoted by Particle Data 38] appears to be still valid. There is a hope that the error could be reduced by a factor of 2 to 4 in the coming years both due to the theory [12, 44] and the recent CLEO measurements of the exclusive semileptonic decays $B \rightarrow(\pi, \varrho) l \nu_{l}$ 45.

\subsection{3 $\left|V_{u b} / V_{c b}\right|$ and $\left|V_{c b}\right|$}

The values in (43) and (44) are not correlated with each other. On the other hand such a correlation is present in the analysis of the CP violating parameter $\varepsilon_{K}$ which is roughly proportional to the fourth power of $\left|V_{c b}\right|$ and linear in $\left|V_{u b} / V_{c b}\right|$. It follows that not all values in (43) and (44) are simultaneously consistent with the observed value of $\varepsilon_{K}$. This is indirectly seen in [46] and has been more explicitly emphasized this year by Herrlich and Nierste [26] and in [15]. Updating and rewriting the analytic lower bound on $m_{t}$ from $\varepsilon_{K}$ [46] one finds 15

$$
\left|\frac{V_{u b}}{V_{c b}}\right|_{\min }=\frac{0.225}{B_{K} A^{2}\left(2 x_{t}^{0.76} A^{2}+1.4\right)}
$$


This bound is shown as a function of $\left|V_{c b}\right|$ for different values of $B_{K}$ and $m_{t}=$ $185 \mathrm{GeV}$ in fig.3. We observe that simultaneously small values of $\left|V_{u b} / V_{c b}\right|$ and $\left|V_{c b}\right|$ although still consistent with (43) and (44), are not allowed by the size of the indirect CP violation observed in $K \rightarrow \pi \pi$.

Fig. 3

\subsection{4 $\eta_{i}$ and $\eta_{B}$}

The NLO values for $\eta_{2}$ and $\eta_{B}$ have been known already for some time [25]:

$$
\eta_{2}=0.57 \pm 0.01 \quad \eta_{B}=0.55 \pm 0.01
$$

The NLO values for $\eta_{1}$ and $\eta_{3}$ are relatively new with the calculation of $\eta_{3}$ completed by Herrlich and Nierste this summer [24, 26]:

$$
\eta_{1}=1.38 \pm 0.20 \quad \eta_{3}=0.47 \pm 0.04
$$

The quoted errors reflect the remaining theoretical uncertainties due to $\Lambda_{\overline{M S}}$ and the quark masses. The references to the leading order calculations can be found in [15].

\subsection{5 $\quad B_{K}$}

Concerning the parameter $B_{K}$, the most recent analyses using the lattice methods [47, 48] $\left(B_{K}=0.83 \pm 0.03\right)$ and the $1 / N$ approach of [49] modified somewhat in [50] give results in the ball park of the $1 / N$ result $B_{K}=0.70 \pm 0.10$ obtained a long time ago 
[49]. In particular the analysis of Bijnens and Prades [50] seems to have explained the difference between these values for $B_{K}$ and the lower values obtained by using the QCD Hadronic Duality approach [51] $\left(B_{K}=0.39 \pm 0.10\right)$ or using the $S U(3)$ symmetry and $\operatorname{PCAC}\left(B_{K}=1 / 3\right)$ [52]. This is gratifying because such low values for $B_{K}$ would require $m_{t}>250 \mathrm{GeV}$ in order to explain the experimental value of $\varepsilon_{K}$ [46, 37, 26]. In our numerical analysis we will use

$$
B_{K}=0.75 \pm 0.15
$$

\subsection{6 $\quad F_{B}$}

There is a vast literature on the lattice calculations of $F_{B}$. The most recent results are somewhat lower than quoted a few years ago. Based on a review by Chris Sachrajda [53], the recent extensive study by Duncan et al. [54 and the analyses in [55] we conclude: $F_{B_{d}}=(180 \pm 40) \mathrm{MeV}$. This together with the earlier result of the European

Collaboration for $B_{B_{d}}$, gives $F_{B_{d}} \sqrt{B_{B_{d}}}=195 \pm 45 \mathrm{MeV}$. The reduction of the error in this important quantity is desirable. These results for $F_{B}$ are compatible with the results obtained using QCD sum rules (e.g.[56]). An interesting upper bound $F_{B_{d}}<195 \mathrm{MeV}$ using QCD dispersion relations has also recently been obtained [57]. In our numerical analysis we will use:

$$
F_{B_{d}} \sqrt{B_{B_{d}}}=200 \pm 40 \mathrm{MeV} .
$$

At this point let us recall that the measurement of $B_{d}^{o}-\bar{B}_{d}^{o}$ mixing parametrized by $x_{d}$ together with $B_{s}^{o}-\bar{B}_{s}^{o}$ mixing parametrized by $x_{s}$ allows to determine $R_{t}$ :

$$
R_{t}=\frac{1}{\sqrt{R_{d s}}} \sqrt{\frac{x_{d}}{x_{s}}} \frac{1}{\lambda} \quad R_{d s}=\frac{\tau_{B_{d}}}{\tau_{B_{s}}} \cdot \frac{m_{B_{d}}}{m_{B_{s}}}\left[\frac{F_{B_{d}} \sqrt{B_{B_{d}}}}{F_{B_{s}} \sqrt{B_{B_{s}}}}\right]^{2}
$$

where $R_{d s}$ summarizes $\mathrm{SU}(3)$-flavour breaking effects. Note that $m_{t}$ and $V_{c b}$ dependences have been eliminated this way and that $R_{d s}$ contains much smaller theoretical uncertainties than the hadronic matrix elements in $x_{d}$ and $x_{s}$ separately. Provided $x_{d} / x_{s}$ has been accurately measured a determination of $R_{t}$ within $\pm 10 \%$ should be possible. Indeed the most recent lattice result [54] gives $F_{B_{s}} / F_{B_{d}}=1.22 \pm 0.04$. A similar result has been obtained using QCD sum rules [58]. It would be useful to know $B_{B_{s}} / B_{B_{d}}$ with similar precision. For $B_{B_{s}}=B_{B_{d}}$ I find $R_{d s}=0.66 \pm 0.07$.

\subsection{7 $\tau_{B}$ and $x_{d}$}

At this meeting the world averages for B-life-times have been presented by Giuliana Rizzo [59]:

$$
\tau\left(B_{d}\right)=(1.57 \pm 0.05) p s \quad \tau\left(B^{+}\right)=(1.63 \pm 0.05) p s \quad \tau\left(B_{s}\right)=(1.58 \pm 0.10) p s
$$

On the other hand the values for $x_{d}$ and $x_{s}$ have been summarized by Achille Stocchi [60]:

$$
x_{d}=0.714 \pm 0.043 \quad x_{s}>8.3(95 \% \text { C.L. })
$$


This is compatible with $x_{d}=0.73 \pm 0.04$ of Aleksan [61]. In what follows I will use

$$
\tau\left(B_{d}\right)=\tau\left(B_{s}\right)=1.6 \text { ps } \quad x_{d}=0.75 \pm 0.06
$$

which is compatible with the above values. I prefer to use $x_{d}$ instead of $\Delta M$ because $x_{d}$ is dimensionless.

\subsection{8 $m_{t}$}

Finally it is important to stress that the discovery of the top quark [39, 40] and its mass measurement had an important impact on the field of rare decays and CP violation reducing considerably one potential uncertainty. As we stated above, in loop induced $\mathrm{B}$ decays the relevant mass parameter is the running current quark mass. In this review we will simply denote this mass by $m_{t}$ and use:

$$
m_{t} \equiv \bar{m}_{t}\left(m_{t}\right)=(170 \pm 15) G e V
$$

Fig. 4

\section{5 "Future" Input Parameters}

The "present" input parameters for the standard analysis are summarized above. It is expected that the future will bring the reduction of errors in the input parameters. For this reason I will also present the results using: 


\begin{tabular}{|c||c||c|}
\hline & Present & Future \\
\hline$\left|V_{t d}\right| / 10^{-3}$ & $6.7-11.9$ & $7.8-9.4$ \\
\hline$\left|V_{t s} / V_{c b}\right|$ & $0.959-0.993$ & $0.975-0.986$ \\
\hline $\sin (2 \beta)$ & $0.33-0.80$ & $0.57-0.73$ \\
\hline $\sin (2 \alpha)$ & $-0.86-1.0$ & $-0.30-0.73$ \\
\hline $\sin (\gamma)$ & $0.61-1.0$ & $0.85-1.0$ \\
\hline$x_{s}$ & $11.3-46.7$ & $19.5-29.4$ \\
\hline
\end{tabular}

Table 2: Output for "present" and "future" inputs.

$$
\begin{aligned}
\left|V_{c b}\right| & =0.040 \pm 0.001 & \left|V_{u b} / V_{c b}\right| & =0.08 \pm 0.01 \\
B_{K} & =0.75 \pm 0.05 & \sqrt{B_{B_{d}}} F_{B_{d}} & =(200 \pm 10) \mathrm{MeV} \\
x_{d} & =0.75 \pm 0.03 & m_{t} & =(170 \pm 5) \mathrm{GeV}
\end{aligned}
$$

with all other input parameters unchanged. It is plausible that these reduced errors will be achieved at the end of this decade, although the central values are not guaranteed.

\subsection{Output of a Standard Analysis}

In table 2 I show the ranges for various quantities of interest which have been found using the sets of input parameters just discussed. This analysis has been done in collaboration with Gerhard Buchalla and Markus Lautenbacher. More details and more results can be found in [15]. In fig. $4 \mathrm{I}$ show the present (a) and the future (b) ranges for the upper corner A of the UT. The solid lines correspond to $R_{t}$ from (50) using $R_{d s}=0.66$ and $x_{s}=10,15,25$ and 40 , respectively. The allowed region has a typical "banana" shape which can be found in many other analyses [37, 62, 26, 63, 64]. The size of the banana and its position depends on the assumed input parameters and on the error analysis which varies from paper to paper. The results in fig. 4 correspond to a simple independent scanning of all parameters within one standard deviation. Effectively such an approach is more conservative than using Gaussian distributions as done in some papers quoted above. Since all these results are selfexplanatory, let me move to other topics.

\section{Three Topics}

\subsection{NLO Corrections to $B_{S L}$}

An important issue are the NLO corrections to the non-leptonic width for B-Mesons which is relevant for the theoretical prediction of the inclusive semileptonic branching 
ratio:

$$
B_{S L}=\frac{\Gamma(B \rightarrow X e \nu)}{\Gamma_{S L}(B)+\Gamma_{N L}(B)}
$$

This calculation can be done within the spectator model corrected for small nonperturbative corrections [9] and more important gluon bremsstrahlung and virtual gluon corrections. The calculation of NLO QCD corrections to $\Gamma_{N L}(B)$ has been done by Altarelli et al. [13] in the DRED renormalization scheme and by Buchalla [21] in the HV scheme neglecting the masses of the final quarks. The results of these papers agree with each other.

It is well known that the inclusion of QCD corrections in the spectator model lowers $B_{S L}$ which otherwise would be roughly $16 \%$. Unfortunately the theoretical branching ratio based on the QCD calculation of refs. [13, 21] turns out to be typically $B_{S L}=$ $(12.5-13.5) \%$ [65] whereas the experimental world average [66] is

$$
B_{S L}^{e x p}=(10.4 \pm 0.4) \%
$$

The inclusion of the leading non-perturbative correction $\mathcal{O}\left(1 / m_{b}^{2}\right)$ lowers slightly the theoretical prediction but gives only $\Delta_{N P} B_{S L}=-0.2 \%$ [9]. On the other hand mass effects in the QCD corrections to $B_{S L}$ seem to play an important role. Bagan et al. [22] using partially the results of Hokim and Pham [67] have demonstrated that the inclusion of mass effects in the QCD calculations of refs. [13, 21] (in particular in the decay $b \rightarrow c \bar{c} s$ (see also 68]) and taking into account various renormalization scale uncertainties improves the situation considerably. Bagan et al. find [22]:

$$
B_{S L}=(12.0 \pm 1.4) \% \quad \text { and } \quad \bar{B}_{S L}=(11.2 \pm 1.7) \%
$$

for the pole quark masses and $\overline{M S}$ masses respectively. Within existing uncertainties, this result does not disagree significantly with the experimental value, although it is still somewhat on the high side.

\subsection{The $n_{c}$ Problem}

The number of charmed hadrons per $\mathrm{B}$ decay, $n_{c}$, is measured as [66]:

$$
n_{c}=1.10 \pm 0.06
$$

There appears to be a conflict between (57) and (59) as stressed by many authors [69, 22, 68]. Indeed the explanation of (57) requires substantial rate for $b \rightarrow c \bar{c} s$ which in turn implies $n_{c} \approx 1.3$. Until recently the error on the predicted $n_{c}$ was too large to draw any definite conclusions. However recently, in an interesting analysis, Buchalla, Dunietz and Yamamoto [70] have considerably improved this estimate in a hybrid approach which combines reliable theoretical calculations with well measured quantities from experiment. They find

$$
n_{c}=1.30 \pm 0.05
$$




\begin{tabular}{|c||c|}
\hline Deep Inelastic Scattering & Non-Leptonic Decays \\
\hline \hline Bjorken Scaling & Factorization \\
\hline Scaling Violations (QCD Logs) & Factorization Breakdown through $\mu$ \\
\hline Scaling Violations (Higher Twists) & Final State Interactions \\
\hline
\end{tabular}

Table 3: Analogy between DIS and Non-Leptonic Decays.

which is significantly larger than the experimental value in (59). They give arguments why the current experimental value of $n_{c}$ is underestimated and expect an increase in the measured $n_{c}$ in the future. It will be interesting to see whether this will turn out to be the case.

\subsection{Factorization}

An important issue is the question of factorization in non-leptonic B-decays. This topic has been extensively discussed by Reinhold Rückl [71] at this workshop and I will only make a few remarks here.

In the strict factorization approach the matrix elements of the operators $Q_{i}$ in (4) are factored into the product of matrix elements of quark currents. Since the resulting matrix elements of $Q_{i}$ are then $\mu$ independent they cannot cancel the $\mu$ dependence of the Wilson coefficients which in the factorization approch are given by effective coefficients $a_{i}(\mu)$ [72]. The full amplitude depends then on $\mu$ which is unphysical. This $\mu$ dependence can only be cancelled by non-factorizable contributions in the matrix elements. One can try to argue that there is some "physical" value of $\mu$ at which factorization takes place. The problem is that the $a_{i}(\mu)$ depend generally also on the renormalization scheme, in particular on the treatment of $\gamma_{5}$ in $D \neq 4$ dimensions. This dependence can again be cancelled only by non-factorizable contributions. A numerical analysis of the $\mu$ and scheme dependence of $a_{i}(\mu)$ can be found in [73. It has been found that $a_{1}(\mu)$ is almost independent both of $\mu$ and the renormalization scheme indicating that the factorization in so-called class 1 decays ("external W-exchange") could afterall be a good approximation. This is in fact the class for which some plausible arguments for factorization can be given [74]. In the case of $a_{2}(\mu)$ the $\mu$ and scheme dependences are found to be very large implying the importance of non-factorizable contributions in class 2 decays to which $B_{d} \rightarrow \psi K_{S}$ belongs. This can be demonstrated using QCD sum rules as discussed by Rückl in these proceedings.

One should stress that there are still other non-factorizable contributions like final state interactions. In a sense there is here some analogy to deep-inelastic scattering. I show this in table 3. Related issues are discussed by Rückl. In any case studies of factorization and in particular the searches for patterns of factorization breakdown in 
non-leptonic decays are very important and should be pursued.

\section{$5 \quad$ Rare B Decays}

\section{$5.1 \quad B \rightarrow X_{s} \gamma$}

The rare decay $B \rightarrow X_{s} \gamma$ plays an important role in the present day phenomenology. It originates from the magnetic $\gamma$-penguin of fig. 1d. The effective hamiltonian for $B \rightarrow X_{s} \gamma$ at scales $\mu=O\left(m_{b}\right)$ is given by

$$
\mathcal{H}_{e f f}(b \rightarrow s \gamma)=-\frac{G_{F}}{\sqrt{2}} V_{t s}^{*} V_{t b}\left[\sum_{i=1}^{6} C_{i}(\mu) Q_{i}+C_{7 \gamma}(\mu) Q_{7 \gamma}+C_{8 G}(\mu) Q_{8 G}\right]
$$

where in view of $\left|V_{u s}^{*} V_{u b} / V_{t s}^{*} V_{t b}\right|<0.02$ we have neglected the term proportional to $V_{u s}^{*} V_{u b}$. The magnetic $\gamma$-penguin is represented here by the operator $Q_{7 \gamma}$.

The complicated structure of (61) makes it clear that representing this decay simply by the diagram in fig.1d misrepresents the true situation. Indeed the perturbative QCD effects involving in particular the operator $Q_{2}$ are very important in this decay. They are known [75, 76] to enhance $B \rightarrow X_{s} \gamma$ in the SM by 2-3 times, depending on the top quark mass. Since the first analyses in [75, 76] a lot of progress has been made in calculating the QCD effects beginning with the work in [77, 78. We will briefly summarize this progress.

A peculiar feature of the renormalization group analysis in $B \rightarrow X_{s} \gamma$ is that the mixing under infinite renormalization between the set $\left(Q_{1} \ldots Q_{6}\right)$ and the operators $\left(Q_{7 \gamma}, Q_{8 G}\right)$ vanishes at the one-loop level. Consequently in order to calculate the coefficients $C_{7 \gamma}(\mu)$ and $C_{8 G}(\mu)$ in the leading logarithmic approximation, two-loop calculations of $\mathcal{O}\left(e g_{s}^{2}\right)$ and $\mathcal{O}\left(g_{s}^{3}\right)$ are necessary. The corresponding NLO analysis requires the evaluation of the mixing in question at the three-loop level.

At present, the coefficients $C_{7 \gamma}$ and $C_{8 G}$ are only known in the leading logarithmic approximation. However the peculiar feature of this decay mentioned above caused that the first fully correct calculation of the leading anomalous dimension matrix has been obtained only in 1993 [79, 80]. It has been confirmed subsequently in [81, 82, 32]. The NLO corrections are only partly known. The two-loop mixing involving the operators $Q_{1} \ldots Q_{6}$ and the two-loop mixing in the sector $\left(Q_{7 \gamma}, Q_{8 G}\right)$ has been calculated in 13, 14, 16, 2, 18, 19] and [20] respectively. The calculation of the three loop mixing between the set $\left(Q_{1} \ldots Q_{6}\right)$ and the operators $\left(Q_{7 \gamma}, Q_{8 G}\right)$ has not be done. The $O\left(\alpha_{s}\right)$ corrections to $C_{7 \gamma}\left(M_{W}\right)$ and $C_{8 G}\left(M_{W}\right)$ have been considered in [83]. Gluon corrections to the matrix elements of magnetic penguin operators have been calculated in [84, 85].

The leading logarithmic calculations $[77,80,81,32,84,86$ can be summarized in a compact form as follows:

$$
\frac{B r\left(B \rightarrow X_{s} \gamma\right)}{B r\left(B \rightarrow X_{c} e \bar{\nu}_{e}\right)}=\frac{\left|V_{t s}^{*} V_{t b}\right|^{2}}{\left|V_{c b}\right|^{2}} \frac{6 \alpha_{Q E D}}{\pi f(z)}\left|C_{7 \gamma}^{(0) e f f}(\mu)\right|^{2}
$$


where $C_{7 \gamma}^{(0) e f f}(\mu)$ is the effective coefficient for which an analytic expression can be found in [86], $z=m_{c} / m_{b}$, and $f(z)$ is the phase space factor in the semileptonic b-decay. The expression given above is based on the spectator model corrected for short-distance QCD effects. Support for this approximation comes from the $1 / m_{b}$ expansions. Indeed the spectator model has been shown to correspond to the leading order approximation in the $1 / m_{b}$ expansion. The next corrections appear at the $\mathcal{O}\left(1 / m_{b}^{2}\right)$ level. The latter terms have been studied by several authors [7, 8, 9] with the result that they affect $\operatorname{Br}\left(B \rightarrow X_{s} \gamma\right)$ and $\operatorname{Br}\left(B \rightarrow X_{c} e \bar{\nu}_{e}\right)$ by only a few percent.

A critical analysis of theoretical and experimental uncertainties present in the prediction for $\operatorname{Br}\left(B \rightarrow X_{s} \gamma\right)$ based on the formula (62) has been made in [86] giving

$$
\operatorname{Br}\left(B \rightarrow X_{s} \gamma\right)_{T H}=(2.8 \pm 0.8) \times 10^{-4}
$$

where the error is dominated by the uncertainty in the choice of the renormalization scale $m_{b} / 2<\mu<2 m_{b}$ as first stressed by Ali and Greub [84] and confirmed in [86]. Since $B \rightarrow X_{s} \gamma$ is dominated by QCD effects, it is not surprising that this scale-uncertainty in the leading order is particularly large.

Fig. 5

The $B \rightarrow X_{s} \gamma$ decay has already been measured and as such appears to be the only unquestionable signal of penguin contributions! In 1993 CLEO reported [87] $\operatorname{Br}(B \rightarrow$ $\left.K^{*} \gamma\right)=(4.5 \pm 1.5 \pm 0.9) \times 10^{-5}$. In 1994 the first measurement of the inclusive rate has been presented by CLEO [ 88 :

$$
\operatorname{Br}\left(B \rightarrow X_{s} \gamma\right)=(2.32 \pm 0.57 \pm 0.35) \times 10^{-4}
$$

where the first error is statistical and the second is systematic. This result agrees with (63) very well although the theoretical and experimental errors should be decreased in 
the future in order to reach a definite conclusion and to see whether some contributions beyond the standard model such as present in the Two-Higgs-Doublet Model (2HDM) or in the Minimal Supersymmetric Standard Model (MSSM) are required. In any case the agreement of the theory with data is consistent with the large QCD enhancement of $B \rightarrow X_{s} \gamma$. Without this enhancement the theoretical prediction would be at least by a factor of 2 below the data.

Fig. 5 presents the SM prediction for the inclusive $B \rightarrow X_{s} \gamma$ branching ratio including the uncertainties discussed in [86] together with the CLEO results represented by the shaded regions. We stress that the theoretical result (the error bars) has been obtained prior to the experimental result. Since the theoretical error is dominated by scale ambiguities a complete NLO analysis is very desirable. Such a complete next-toleading calculation of $B \rightarrow X_{s} \gamma$ is described in [86] in general terms. As demonstrated formally there the cancellation of the dominant $\mu$-dependence in the leading order can then be achieved.

In this connection we would like to mention the analysis of [89] in which the known two-loop mixing in the sector $\left(Q_{1} \ldots Q_{6}\right)$ (see table 1 ) has been added to the leading order analysis of $B \rightarrow X_{s} \gamma$. Strong renormalization scheme dependence of the resulting branching ratio has been found, giving the branching ratio $(1.7 \pm 0.2) \cdot 10^{-4}$ and $(2.3 \pm$ $0.3) \cdot 10^{-4}$ at $\mu=5 \mathrm{GeV}$ for $\mathrm{HV}$ and NDR schemes respectively. This strong scheme dependence in the partial NLO analysis presented in 89 demonstrates very clearly the need for a full analysis. In view of this we think that the decrease of the branching ratio for $B \rightarrow X_{s} \gamma$ relative to the LO prediction, found in 89 and given by $\operatorname{Br}(B \rightarrow s \gamma)=$ $(1.9 \pm 0.2 \pm 0.5) \cdot 10^{-4}$, is still premature and one should wait until the full NLO analysis has been done.

Finally it should be stressed that in spite of theoretical and experimental uncertainties the CLEO measurement of the inclusive rate provided already now an interesting lower bound on the mass of the charged Higgs in the most popular two Higgs doublet model. At 95\% C.L. CLEO 88 finds $m_{H^{ \pm}}>250 \mathrm{GeV}$ with a similar result obtained recently by Münz 90. More on radiative $\mathrm{B}$ decays can be found in a recent review by Ali 91 .

\section{$5.2 \quad B \rightarrow X_{s} e^{+} e^{-}$Beyond Leading Logarithms}

The rare decay $B \rightarrow X_{s} e^{+} e^{-}$originates from the electroweak penguin diagrams of fig. 1f. The effective hamiltonian for $B \rightarrow X_{s} e^{+} e^{-}$at scales $\mu=O\left(m_{b}\right)$ is given by

$$
\mathcal{H}_{e f f}\left(b \rightarrow s e^{+} e^{-}\right)=\mathcal{H}_{e f f}(b \rightarrow s \gamma)-\frac{G_{F}}{\sqrt{2}} V_{t s}^{*} V_{t b}\left[C_{9 V}(\mu) Q_{9 V}+C_{10 A}\left(M_{W}\right) Q_{10 A}\right]
$$

where we have again neglected the term proportional to $V_{u s}^{*} V_{u b}$ and $\mathcal{H}_{\text {eff }}(b \rightarrow s \gamma)$ is given in (61). In addition to the operators relevant for $B \rightarrow X_{s} \gamma$, there are two new operators $Q_{9 V}$ and $Q_{10 A}$ which are generated through the electroweak penguin diagrams of fig. If and the related box diagrams needed mainly to keep gauge invariance. There 
is a large literature on this dacay. In particular Hou et al. [92] stressed the strong dependence of $B \rightarrow X_{s} e^{+} e^{-}$on $m_{t}$. Further references to phenomenology can be found in [33. Here we concentrate on QCD corrections.

The QCD corrections to $B \rightarrow X_{s} e^{+} e^{-}$have been calculated over the last years with increasing precision by several groups 93, 94, 95, 32 culminating in two complete nextto-leading QCD calculations [32, 33] which agree with each other. An important gain due to these NLO calculations is a considerable reduction in the $\mu$-dependence of the resulting branching ratio. Whereas in $\mathrm{LO}$ an uncertainty as large as $\pm 20 \%$ can be found, it is reduced as shown in [33 below $\pm 8 \%$ after the inclusion of NLO corrections. This is very encouraging for the analogous efforts in $B \rightarrow X_{s} \gamma$ as discussed above.

An extensive numerical analysis of the differential decay rate including NLO corrections has been presented in [33]. As an example we show in fig. 6 the differential decay rate $R(\hat{s})$ divided by $\Gamma\left(B \rightarrow X_{c} e \bar{\nu}\right)$ as a function of $\hat{s}=\left(p_{e^{+}}+p_{e^{-}}\right)^{2} / m_{b}^{2}$ for $m_{t}=170 \mathrm{GeV}$ and $\Lambda \frac{(5)}{M S}=225 \mathrm{MeV}$. We observe that the QCD suppression in the leading order [93] is substantially weakened by the inclusion of NLO corrections. The same result has been obtained by Misiak [32]. The $1 / m_{b}^{2}$ corrections calculated in [96] enhance these results by roughly $10 \%$.

Fig. 6

Finally I would like to refer to some recent papers [97], where other aspects of $B \rightarrow X_{s} e^{+} e^{-}$have been discussed and where further references can be found. 


\section{3 $B \rightarrow \mu \bar{\mu}$ and $B \rightarrow X_{s} \nu \bar{\nu}$}

$B \rightarrow \mu \bar{\mu}$ and $B \rightarrow X_{s} \nu \bar{\nu}$ are the theoretically cleanest decays in the field of rare Bdecays. $B \rightarrow \mu \bar{\mu}$ and $B \rightarrow X_{s} \nu \bar{\nu}$ are dominated by the $Z^{0}$-penguin and box diagrams involving top quark exchanges. Their $m_{t}$-dependence is fully described by the squares of $Y\left(x_{t}\right)$ and $X\left(x_{t}\right)$ in (23) and (22) respectively.

The NLO corrections to both decays have been calculated by Buchalla and myself [28]. These calculations reduced considerably the theoretical uncertainties in the branching ratios related to the scale $\mu_{t}$ present in $\bar{m}_{t}\left(\mu_{t}\right)$. As a by-product of this work we have also pointed out that the previously published branching ratios $\operatorname{Br}\left(B_{s} \rightarrow \mu \bar{\mu}\right)$ missed an overall factor of 2 which clearly has important phenomenological implications.

I will discuss here only $B_{s} \rightarrow \mu \bar{\mu}$. Choosing $\mu_{t}=m_{t}$ the final expression for $B_{s} \rightarrow \mu \bar{\mu}$ including NLO corrections takes a particularly simple form [28]. Updating the input parameters one has [15]:

$$
\operatorname{Br}\left(B_{s} \rightarrow \mu \bar{\mu}\right)=4.18 \cdot 10^{-9}\left[\frac{F_{B_{s}}}{230 \mathrm{MeV}}\right]^{2}\left[\frac{\bar{m}_{t}\left(m_{t}\right)}{170 \mathrm{GeV}}\right]^{3.12}\left[\frac{\left|V_{t s}\right|}{0.040}\right]^{2}\left[\frac{\tau_{B_{s}}}{1.6 p s}\right]
$$

The impact of NLO calculations is best illustrated by giving the theoretical uncertainty due to the choice of $\mu_{t}$ in the leading order and after the inclusion of the next-to-leading corrections:

$$
\operatorname{Br}\left(B_{s} \rightarrow \mu \bar{\mu}\right)=(4.00 \pm 0.50) \cdot 10^{-9} \quad \Rightarrow \quad(4.10 \pm 0.05) \cdot 10^{-9}
$$

The reduction of the theoretical uncertainty is truly impressive.

Next I would like to quote the standard model expectation for $\operatorname{Br}\left(B_{s} \rightarrow \mu \bar{\mu}\right)$ based on the input parameters collected in sect. 3.4 and $F_{B_{s}}=230 \pm 40 \mathrm{MeV}$. We find [15]

$$
1.7 \cdot 10^{-9} \leq \operatorname{Br}\left(B_{s} \rightarrow \mu \bar{\mu}\right) \leq 8.4 \cdot 10^{-9}
$$

Taking on the other hand "future" input of (55) and $F_{B_{s}}=230 \pm 10 \mathrm{MeV}$ gives a smaller range:

$$
3.1 \cdot 10^{-9} \leq \operatorname{Br}\left(B_{s} \rightarrow \mu \bar{\mu}\right) \leq 5.0 \cdot 10^{-9}
$$

Finally I would like to stress several virtues of this very interesting decay:

- The strong dominance of the internal top exchanges and a short distance character of the contributions allows in contrast to $B \rightarrow X_{d} \gamma$ a clean determination of $\left|V_{t d}\right|$ by measuring the ratio of $\operatorname{Br}\left(B_{d} \rightarrow \mu \bar{\mu}\right)$ and $\operatorname{Br}\left(B_{s} \rightarrow \mu \bar{\mu}\right)$

- In conjunction with a future measurement of $x_{s}$, the branching ratio $\operatorname{Br}\left(B_{s} \rightarrow \mu \bar{\mu}\right)$ could help to determine the non-perturbative parameter $B_{B_{s}}$ and consequently allow a test of existing non-perturbative methods:

$$
B_{B_{s}}=\left[\frac{x_{s}}{22.1}\right]\left[\frac{\bar{m}_{t}\left(m_{t}\right)}{170 \mathrm{GeV}}\right]^{1.6}\left[\frac{4.2 \cdot 10^{-9}}{\operatorname{Br}\left(B_{s} \rightarrow \mu \bar{\mu}\right)}\right]
$$


- $B_{s} \rightarrow \mu \bar{\mu}$ being theoretically very clean is very suitable for tests of physics beyond the standard model

All efforts should be made to measure this decay. The experimental prospects are discussed in other talks in these proceedings.

\section{Express Review of CP Violation}

\subsection{Preliminaries}

$\mathrm{CP}$ violation in $\mathrm{B}$ decays is certainly one of the most important targets of $\mathrm{B}$ factories and of dedicated $\mathrm{B}$ experiments at hadron facilities. It is well known that $\mathrm{CP}$ violating effects are expected to occur in a large number of channels at a level attainable at forthcoming experiments. Moreover there exist channels which offer the determination of CKM phases essentially without any hadronic uncertainties. Since CP violation in B decays has been already reviewed in a special talk by Michael Gronau 98 at this meeting and since in addition extensive reviews can be found in the literature [99], let me concentrate only on the most important points.

\subsection{Strategies for $(\alpha, \beta, \gamma)$}

During the last 15 years many efforts have been made to find means of measuring the angles in the unitarity triangle of fig. 2 without any hadronic uncertainties. The main strategies are as follows:

\subsection{1 $\quad B^{0}$-Decays to CP Eigenstates}

A time dependent asymmetry in the decay $B^{0} \rightarrow f$ with $f$ being a $\mathrm{CP}$ eigenstate is given by

$$
a_{C P}(t, f)=\mathcal{A}_{C P}^{\text {dir }}(B \rightarrow f) \cos (\Delta M t)+\mathcal{A}_{C P}^{\text {mix-ind }}(B \rightarrow f) \sin (\Delta M t)
$$

where we have separated the direct CP-violating contributions from those describing mixing-induced $\mathrm{CP}$ violation:

$$
\mathcal{A}_{C P}^{\text {dir }}(B \rightarrow f) \equiv \frac{1-\left|\xi_{f}\right|^{2}}{1+\left|\xi_{f}\right|^{2}}, \quad \mathcal{A}_{C P}^{\operatorname{mix}-i n d}(B \rightarrow f) \equiv \frac{2 \operatorname{Im} \xi_{f}}{1+\left|\xi_{f}\right|^{2}}
$$

In (71), $\Delta M$ denotes the mass splitting of the physical $B^{0}-\bar{B}^{0}-$ mixing eigenstates. The quantity $\xi_{f}$ containing essentially all the information needed to evaluate the asymmetries (72) is given by

$$
\xi_{f}=\exp \left(-i \phi_{M}\right) \frac{A(\bar{B} \rightarrow f)}{A(B \rightarrow f)}
$$


with $\phi_{M}$ denoting the weak phase in the $B-\bar{B}$ mixing and $A(B \rightarrow f)$ the decay amplitude.

Generally several decay mechanisms with different weak and strong phases can contribute to $A(B \rightarrow f)$. These are tree diagram (current-current) contributions, QCD penguin contributions and electroweak penguin contributions. If they contribute with similar strength to a given decay amplitude the resulting CP asymmetries suffer from hadronic uncertainies related to matrix elements of the relevant operators $Q_{i}$.

An interesting case arises when a single mechanism dominates the decay amplitude or the contributing mechanisms have the same weak phases. Then

$$
\xi_{f}=\exp \left(-i \phi_{M}\right) \exp \left(i 2 \phi_{D}\right), \quad\left|\xi_{f}\right|^{2}=1
$$

where $\phi_{D}$ is the weak phase in the decay amplitude. In this particular case the hadronic matrix elements drop out, the direct $\mathrm{CP}$ violating contribution vanishes and the mixinginduced CP asymmetry is given entirely in terms of the weak phases $\phi_{M}$ and $\phi_{D}$. In particular the time integrated asymmetry is given by

$$
a_{C P}(f)= \pm \sin \left(2 \phi_{D}-\phi_{M}\right) \frac{x_{d, s}}{1+x_{d, s}^{2}}
$$

where \pm refers to $f$ being a $C P= \pm$ eigenstate and $x_{d, s}$ are the $B^{0}-\bar{B}^{0}$ mixing parameters. Then one finds for $B_{d} \rightarrow \psi K_{S}$ and $B_{d} \rightarrow \pi^{+} \pi^{-}$

$$
a_{C P}\left(\psi K_{S}\right)=-\sin (2 \beta) \frac{x_{d}}{1+x_{d}^{2}} \quad a_{C P}\left(\pi^{+} \pi^{-}\right)=-\sin (2 \alpha) \frac{x_{d}}{1+x_{d}^{2}}
$$

where we have neglected for a moment QCD penguins in $a_{C P}\left(\pi^{+} \pi^{-}\right)$. Since in the usual unitarity triangle one side is known, it suffices to measure two angles to determine the triangle completely. This means that the measurements of $\sin 2 \alpha$ and $\sin 2 \beta$ can determine the parameters $\varrho$ and $\eta$. The main virtues of this determination are as follows:

- No hadronic or $\Lambda_{\overline{M S}}$ uncertainties.

- No dependence on $m_{t}$ and $V_{c b}$ (or $A$ ).

Unfortunately life is not so easy and there are only a few channels for which this fortunate situation takes place. In addition studies of this type require tagging ( distinction between unmixed $B^{0}$ and $\bar{B}^{0}$ at $t=0$ ) as well as two time dependent rates $B^{0}(t) \rightarrow f$ and $\bar{B}^{0}(t) \rightarrow f$. Both tagging and time dependent studies are certainly not easy.

\subsubsection{Decays to CP-Non-Eigenstates}

One can of course study also decays to CP non-eigenstates. This, in addition to tagging, requires generally four time dependent rates $B^{0}(t) \rightarrow f, \bar{B}^{0}(t) \rightarrow f, B^{0}(t) \rightarrow \bar{f}$ and $\bar{B}^{0}(t) \rightarrow \bar{f}[100$. In certain cases this approach gives interesting results. 


\subsubsection{Triangle Constructions}

Here one attempts to extract $\alpha, \beta$, and $\gamma$ from branching ratios only. Neither tagging nor time-dependent measurements are needed. On the other hand these methods require measurements of several branching ratios in order to eliminate the hadronic uncertainties. The prototypes of such studies are the method of Gronau and Wyler 101 and the method of Gronau and London [102] with the latter using the $S U(2)$-flavour (isospin) symmetry. More recently methods based on SU(3)-triangle relations have been proposed [103, 104, 105. I will return to them below.

\subsection{Theoretically Clean Determinations of $(\alpha, \beta, \gamma)$}

In my opinion there exist only five determinations of $(\alpha, \beta, \gamma)$ in B decays which fully deserve the name "theoretically clean". Let me discuss them briefly one-by-one.

\subsection{1 $\quad B_{d} \rightarrow \psi K_{S}$ and $\beta$}

The mixing induced CP-asymmetry in this "gold-plated" decay allows in the standard model a direct measurement of the angle $\beta$ as pointed out by Bigi and Sanda 106 a long time ago. In this decay the QCD penguins and EW penguins have to an excellent approximation the same weak phases as the leading tree contributions. This results in the formula given in (76) which offers a clean determination of $\beta$.

\subsection{2 $\quad B_{d} \rightarrow \pi^{+} \pi^{-}$and $\alpha$}

In this case the formula (76) deoes not really apply because of the presence of QCD penguin contributions which have different phases than the leading tree contributions. The asymmetry $a_{C P}\left(\pi^{+} \pi^{-}\right)$measures then $2 \alpha+\theta_{P}$ where the unknown phase $\theta_{P}$ signals the presence of QCD penguins. Using the isospin symmetry and the related triangle construction Gronau and London 102 have demonstrated how the unknown phase $\theta_{P}$ can be found by measuring in addition the branching ratios $\operatorname{Br}\left(B^{+} \rightarrow \pi^{+} \pi^{0}\right), \operatorname{Br}\left(B^{0} \rightarrow\right.$ $\pi^{0} \pi^{0}$ ) and the branching ratios of $\mathrm{CP}$ conjugate channels. With this information the asymmetry $a_{C P}\left(\pi^{+} \pi^{-}\right)$offers a clean determination of $\alpha$. The smallness of $\operatorname{Br}\left(B_{d} \rightarrow\right.$ $\left.\pi^{0} \pi^{0}\right)\left(\leq \mathcal{O}\left(10^{-6}\right)\right.$ [107, 108] is a weak point of this method. It has been pointed out by Deshpande and He [109] that the presence of EW penguins could have a sizable impact on the GL method. A closer look shows, however that this impact is rather small [110, at most a few \%. Moreover as I will discuss below a method has been proposed to estimate this effect quantitatively [111, 112].

\subsection{3 $\quad B^{ \pm} \rightarrow D_{C P}^{0} K^{ \pm}$and $\gamma$}

This decay involves only tree diagram contributions and requires six decay rates $B^{ \pm} \rightarrow$ $D_{C P}^{0} K^{ \pm}, B^{+} \rightarrow D^{0} K^{+}, \bar{D}^{0} K^{+}$and $B^{-} \rightarrow D^{0} K^{-}, \bar{D}^{0} K^{-}$. A known triangle construction due to Gronau and Wyler [101] allows then a clean determination of $\gamma$. The 
virtue of this method is that neither tagging nor time-dependent studies are required. Moreover the observation of $\mathrm{CP}$ violation in $B^{ \pm} \rightarrow D_{C P}^{0} K^{ \pm}$would signal automatically direct $\mathrm{CP}$ violation. A possible difficulty is the disparity in the size of the expected branching ratios needed to construct the triangles in question. Whereas four branching ratios listed above are expected to be $\mathcal{O}\left(10^{-4}-10^{-5}\right)$, the branching ratio of the colour supressed channels $B^{ \pm} \rightarrow D^{0} K^{ \pm}$are expected to be by one order of magnitude smaller. On one hand such a small branching ratio is difficult to measure. On the other hand the resulting triangles will have one side very small making the extraction of $\gamma$ difficult. A similar method using neutral B-decays has been proposed by Dunietz [113.

\subsection{4 $B_{s} \rightarrow D_{s}^{+} K^{-}, \bar{B}_{s} \rightarrow D_{s}^{-} K^{+}$and $\gamma$}

This method suggested by Aleksan, Dunietz and Kayser [114 is unaffected by penguin contributions. A full time dependent analysis allows a clean measurement of $\sin ^{2} \gamma$. Since the expected branching ratio is $\mathcal{O}\left(10^{-4}\right)$, this method is in principle feasible although the expected large $B_{s}^{0}-\bar{B}_{s}^{0}$ mixing makes this measurement very challenging.

\subsection{5 $\quad B_{s} \rightarrow \psi \phi$ and $\eta$}

This is an analog of $B_{d} \rightarrow \psi K_{s}$. In the leading order of the Wolfenstein parametrization the asymmetry $a_{C P}(\psi \phi)$ vanishes. Including higher order terms in $\lambda$ I find

$$
a_{C P}(\psi \phi)=2 \lambda^{2} \eta \frac{x_{s}}{1+x_{s}^{2}}
$$

where $\lambda$ and $\eta$ are the Wolfenstein parameters. This agrees with Dunietz [115] although he expressed this asymmetry in terms of $\gamma$ and some poorly known CKM elements. I think the presentation in (77) is simpler: $a_{C P}(\psi \phi)$ measures $\eta$. With $\lambda=0.22$ and $\eta=0.35$ one has $2 \lambda^{2} \eta=0.03$. The dilution factor suppresses the asymmetry further and clearly the measurement of $\eta$ this way is very challenging. Time-dependent studies at the LHC could however reach the expected level. Moreover the very clean character of this asymmetry and its smallness can also be used in the search for the physics beyond the standard model.

\section{4 $S U(3)$ Triangles and Electroweak Penguins}

\subsubsection{Preliminaries}

Clearly the future B experiments will measure many more than just the clean channels listed above and it is important to get new ideas for other determinations of $\alpha, \beta$ and $\gamma$.

Last year in a series of interesting papers Gronau, Hernandez, London and Rosner (GHLR) 103, 104, 105 used the $S U(3)$ flavour symmetry of strong interactions combined with certain dynamical assumptions (neglect of annihilation diagrams etc.) to 
derive simple relations among B-decay amplitudes into $\pi \pi, \pi K$ and $K \bar{K}$ final states. These $S U(3)$ relations should allow to determine in a clean manner both the weak phases of the CKM matrix and strong final state interaction phases by measuring only branching ratios of the relevant $\mathrm{B}$ decays.

In spite of the attractiveness of this approach, it contains certain limitations. Let me first mention the one discussed in [116]. Irrespectively of the uncertainties related to $\mathrm{SU}(3)$-breaking, discussed in 117, the success of this approach depends in the case of $\beta$ on whether the QCD penguin amplitudes are fully dominated by the diagrams with internal top quark exchanges. As we have shown in 116 this full dominance cannot be justified and the presence of QCD penguin contributions with internal u- and c- quarks precludes a clean determination of the angle $\beta$ by using this approach. On the other hand, as we have shown, the determination of the angle $\gamma$ is unaffected by these new contributions.

Yet there are still other contributions which were not taken into account by GHLR. These are the electroweak penguin contributions. As pointed out by Deshpande and He [109] the EW penguins do have an impact on the GHLR approach even in the case of $\gamma$. Before discussing this let me briefly review the importance of $\mathrm{EW}$ penguins in $\mathrm{B}$ decays in general.

\subsubsection{Electroweak Penguins and B decays}

During the last two years there has been a considerable interest in the role of electroweak penguin contributions in non-leptonic $B$-decays. Since the Wilson coefficients of the corresponding local operators increase strongly with the top-quark mass, it has been found by Robert Fleischer 118, 119, 120 that the role of the electroweak penguins can be substantial in certain decays. This is for instance the case of the decay $B^{-} \rightarrow$ $K^{-} \Phi$ [118, which exhibits sizable electroweak penguin effects. More interestingly, there are even some channels, such as $B^{-} \rightarrow \pi^{-} \Phi$ [119] and $B_{s} \rightarrow \pi^{0} \Phi$ [120, which are dominated completely by electroweak penguin contributions and which should, thus, allow interesting insights into the physics of the corresponding operators. In this respect, the decay $B_{s} \rightarrow \pi^{0} \Phi$ (or similar transitions such as $B_{s} \rightarrow \rho^{0} \Phi$ ) is very promising due to its special isospin-, CKM- and colour-structure [120. As the branching ratio of this mode is expected to be of $\mathcal{O}\left(10^{-7}\right)$, it will unfortunately be rather difficult to analyze this decay experimentally. The electroweak penguin effects discussed in [118, 120] have been confirmed by other authors 1121-123.

In the foreseeable future the branching ratios of $\mathcal{O}\left(10^{-5}\right)$ and possibly $\mathcal{O}\left(10^{-6}\right)$ will be experimentally available and it is important to ask about the role of electroweak penguin effects in the corresponding channels. In particular, the question arises whether the usual strategies for the determination of the CKM-phases are affected by the presence of the electroweak penguin contributions.

As we have stated above the five methods reviewed in section 6.3 are, - except for the Gronau-London method - unaffected by EW penguins. It can also be shown that 
the impact of the $\bar{b} \rightarrow \bar{d}$ EW penguins on the Gronau-London method is small [110, 111]. On the other hand the point of Despande and He [109] that the $\bar{b} \rightarrow \bar{s}$ EW penguins have a considerable impact on the GHLR method is now well accepted.

In this connection GHLR have presented a systematic classification of electroweak penguins in two-body $B$-decays [110]. Moreover, in this paper they have constructed an amplitude quadrangle for $B \rightarrow \pi K$ decays that can be used - at least in principle - to extract the CKM-angle $\gamma$ irrespectively of the presence of electroweak penguins. Unfortunately, from the experimental point of view this approach is rather difficult, because one diagonal of the quadrangle corresponds to the amplitude of the electroweak penguin dominated $B_{s}$-decay $B_{s} \rightarrow \pi^{0} \eta$ which is expected to have a very small branching ratio at the $\mathcal{O}\left(10^{-7}\right)$ level. Another $S U(3)$-symmetry based method of extracting $\gamma$, where electroweak penguins are also eliminated, has been presented by Deshpande and $\mathrm{He}$ [124]. Although this approach using the charged $B$-decays $B^{-} \rightarrow\left\{\pi^{-} \bar{K}^{0}, \pi^{0} K^{-}, \eta K^{-}\right\}$and $B^{-} \rightarrow \pi^{-} \pi^{0}$ should be more promising for experimentalists, it is affected by $\eta-\eta^{\prime}$ mixing and other $S U(3)$-breaking effects and therefore cannot be regarded as a clean measurement of $\gamma$.

\subsubsection{Towards the Control over Electroweak Penguins}

In view of this situation, it would be useful to determine the electroweak penguin contributions experimentally. Once this has been achieved, their role in a variety of $B$-decays could be explicitly found. Although some thoughts on this issue have appeared in [110], no constructive quantitative method has been proposed there.

Here I would like to report on a work of Fleischer and myself [111] in which we have suggested a different "philosophy" of applying the $S U(3)$ amplitude relations. In contrast to GHLR, we think that these relations are more useful from the phenomenological point of view if one uses the phase $\gamma$ as one of the central inputs. As we have stated above, there are already methods on the market allowing a measurement of this phase in an absolutely clean way without any effect coming from the electroweak penguins.

At first sight, this new philosophy might appear not useful because one of the goals of the GHLR strategy was precisely the determination of $\gamma$. Yet, as we have seen, this program is difficult to realize without further inputs. On the other hand, as shown in [111], once the phase $\gamma$ is used as an input, the electroweak penguin contributions can be straightforwardly determined. This knowledge subsequently allows the determination of CKM-phases in a variety of $B$-decays [111, 112]. Consequently, with this new strategy, the GHLR method is resurrected. Moreover, the impact of electroweak penguins on the $\alpha$-determination using $B(\bar{B}) \rightarrow \pi \pi$ decays can be quantitatively estimated.

Our strategy proceeds in three steps. In the first step $\gamma$ is taken form a decay without EW-penguin contributions (see for instance sections 6.3 .3 or 6.3.4). In the second step one measures the branching ratios for $B^{+} \rightarrow \pi^{+} K^{0}, B^{+} \rightarrow \pi^{0} K^{+}, B^{-} \rightarrow \pi^{0} K^{-}$, $B_{d}^{0} \rightarrow \pi^{-} K^{+}$and $\bar{B}_{d}^{0} \rightarrow \pi^{+} K^{-}$and using only $S U(2)$ symmetry finds the $\bar{b} \rightarrow \bar{s}$ EWpenguin. In the third step one takes $\beta$ from a clean decay like $B \rightarrow \psi K_{S}$ and using 
$S U(3)$ symmetry determines the small $\bar{b} \rightarrow \bar{d}$ EW-penguin. Note that in this method only branching ratios $\mathcal{O}\left(10^{-5}\right)$ are required.

Having determined $\bar{b} \rightarrow \bar{s}$ and $\bar{b} \rightarrow \bar{d}$ EW penguins this way one can study their effects in other decays. In particular their effect on the $\alpha$-determination by means of $B \rightarrow \pi \pi$ can be studied quantitatively. More details on this method and an alternative strategy can be found in [11].

\subsubsection{Other Ideas}

The Gronau-London method for the $\alpha$-determination involves the experimentally difficult mode $B_{d} \rightarrow \pi^{0} \pi^{0}$ which is expected to be $\leq \mathcal{O}\left(10^{-6}\right)$ 108. Moreover it is slightly affected by EW penguin contributions. In [107] we have presented an alternative method of determining $\alpha$ by performing simultaneous measurements of the mixing-induced CP asymmetries of the decays $B_{d} \rightarrow \pi^{+} \pi^{-}$and $B_{d} \rightarrow K^{0} \bar{K}^{0}$. The accuracy of this method is limited by $S U(3)$ breaking effects but it is unaffected by $\mathrm{EW}$ penguins. Moreover the decay $B_{d} \rightarrow K^{0} \bar{K}^{0}$ might be easier to measure than $B_{d} \rightarrow \pi^{0} \pi^{0}$. In this connection I would like to mention that in contrast to previous claims the CP asymmetry in $B_{d} \rightarrow K^{0} \bar{K}^{0}$ is non-vanishing in the standard model. Indeed as shown by Fleischer [125] the inclusion of QCD penguins with internal $\mathrm{u}$ - and c-quarks in $B_{d} \rightarrow K^{0} \bar{K}^{0}$ can result in a $\mathrm{CP}$ asymmetry as large as $\mathcal{O}(50 \%)$.

Next I would like to mention a recent interesting paper by Fleischer [112] where various strategies for fixing the angle $\gamma$ and obtaining experimental insight into the world of EW-penguins have been presented. A by-product of this work is a refined estimate of the role of EW-penguins in the $\alpha$-determination by means of $B \rightarrow \pi \pi$ decays.

\section{Classification}

It is probably a good place to group the various decays and quantities into three distinct classes. I include in this classification also K-decays.

\subsection{Class 1}

These are the decays with essentially no theoretical uncertainties:

- $\mathrm{CP}$ violation in $B_{d} \rightarrow \psi K_{S}, B^{ \pm} \rightarrow D_{C P} K^{ \pm}, B_{s} \rightarrow D_{s} K B_{s} \rightarrow \psi \phi, B \rightarrow \pi \pi$,

- Rare B-decays: $B_{d} \rightarrow l \bar{l}, B_{d} \rightarrow X_{s} \nu \bar{\nu}, x_{d} / x_{s}$

- Rare K-decays: $K_{L} \rightarrow \pi^{0} \nu \bar{\nu}, K^{+} \rightarrow \pi^{+} \nu \bar{\nu}$ 


\subsection{Class 2}

Here I group the quantities or decays with moderate theoretical uncertainties which should be considerably reduced in the next five years. In particular I assume that the uncertainties in $B_{K}$ and $F_{B} \sqrt{B}$ will be reduced below $10 \%$ and that the NLO corrections to $B \rightarrow X_{s} \gamma$ will be completed.

- $B \rightarrow X_{s} \gamma, B \rightarrow X_{s} e^{+} e^{-}, B \rightarrow K^{*} e^{+} e^{-}$

- $x_{d}, x_{s},\left|V_{c b}\right|_{e x c l},\left|V_{c b}\right|_{i n c l},\left|V_{u b} / V_{c b}\right|_{\text {incl }}$

- Some CP asymmetries in B-decays discussed in section 6.

- $\varepsilon_{K}$ and $K_{L} \rightarrow \pi^{0} e^{+} e^{-}$

\subsection{Class 3}

Here we have a list of important decays with large theoretical uncertainties which can only be removed by a dramatic progress in non-perturbative techniques:

- $\mathrm{CP}$ asymmetries in most $B^{ \pm}$-decays

- $B_{d} \rightarrow K^{*} \gamma$, Non-leptonic B-decays, $\left|V_{u b} / V_{c b}\right|_{e x c l}$

- $\varepsilon^{\prime} / \varepsilon, K \rightarrow \pi \pi, \Delta M\left(K_{L}-K_{s}\right), K_{L} \rightarrow \mu \bar{\mu}$, hyperon decays and so on.

It should be stressed that even in the presence of theoretical uncertainties a measurement of a non-vanishing ratio $\varepsilon^{\prime} / \varepsilon$ or a non-vanishing CP asymmetry in charged B-decays would signal direct $\mathrm{CP}$ violation excluding superweak scenarios [126]. This is not guaranteed by several clean decays of class 1 [127] except for $B^{ \pm} \rightarrow D_{C P} K^{ \pm}$.

\section{Future Visions}

Let us next concentrate on decays of class 1 which as we have stated above are essentially free of hadronic uncertainties. As we have seen in section 3 using the standard analysis with rather optimistic assumptions about the theoretical and experimental errors it was difficult to achieve the accuracy better than $\Delta \varrho= \pm 0.10$ and $\Delta \eta= \pm 0.05$. Let us then see what can be achieved with the decays of class 1 .

\section{1 $(\rho, \eta)$ from $(\sin (2 \alpha), \sin (2 \beta))$}

With $a \equiv \sin (2 \alpha)$ and $b \equiv \sin (2 \beta)$ one determines $\varrho, \eta$ as follows [128]:

$$
\bar{\varrho}=1-\bar{\eta} r_{+}(b) \quad, \quad \bar{\eta}=\frac{r_{-}(a)+r_{+}(b)}{1+r_{+}^{2}(b)}
$$




\begin{tabular}{|c||c||c|c|c|}
\hline & Central & $I$ & $I I$ & $I I I$ \\
\hline $\sin (2 \alpha)$ & 0.40 & \pm 0.08 & \pm 0.04 & \pm 0.02 \\
\hline $\sin (2 \beta)$ & 0.70 & \pm 0.06 & \pm 0.02 & \pm 0.01 \\
\hline \hline$\varrho$ & 0.072 & \pm 0.040 & \pm 0.016 & \pm 0.008 \\
\hline$\eta$ & 0.389 & \pm 0.044 & \pm 0.016 & \pm 0.008 \\
\hline$\left|V_{u b} / V_{c b}\right|$ & 0.087 & \pm 0.010 & \pm 0.003 & \pm 0.002 \\
\hline
\end{tabular}

Table 4: Determinations of $\varrho$ and $\eta$ using $\sin (2 \alpha)$ and $\sin (2 \beta)$

where

$$
r_{ \pm}(z)=\frac{1}{z}\left(1 \pm \sqrt{1-z^{2}}\right) \quad z=a, b
$$

where $(\bar{\varrho}, \eta)$ are defined in (30). As illustrative examples we consider in table 4 three scenarios. The first two rows give the assumed input parameters and their experimental errors. The remaining rows give the results for $\varrho, \eta$ and $\left|V_{u b} / V_{c b}\right|$. Further results can be found in [128]. The accuracy in the scenario I should be achieved at B-factories and HERA-B. Scenarios II and III correspond potentially to B-physics at Fermilab during the Main Injector era and at LHC respectively.

Table 4 shows very clearly the potential of $\mathrm{CP}$ asymmetries in B-decays in the determination of CKM parameters. It should be stressed that this high accuracy is not only achieved because of our assumptions about future experimental errors in the scenarios considered, but also because $\sin (2 \alpha)$ is a very sensitive function of $\varrho$ and $\eta$ [37], and most importantly because of the clean character of the quantities considered.

\section{$8.2(\rho, \eta)$ from $\left(R_{t}, \sin (2 \beta)\right)$}

An alternative strategy is to use the measured value of $R_{t}$ instead of $\sin (2 \alpha)$. A clean measurement of $R_{t}$ can be achieved using the ratio $x_{d} / x_{s}$. Then (78) is replaced by [129]

$$
\bar{\varrho}=1-\bar{\eta} r_{+}(b) \quad, \quad \bar{\eta}=\frac{R_{t}}{\sqrt{2}} \sqrt{b r_{-}(b)}
$$

The result of this exercise is shown in table 5. Although this determination of CKM parameters cannot fully compete with the previous one the consistency of both determinations will offer an important test of the standard model.

\section{$8.3 \sin (2 \beta)$ from $K \rightarrow \pi \nu \bar{\nu}$}

It has been pointed out in 130] that measurements of $\operatorname{Br}\left(K^{+} \rightarrow \pi^{+} \nu \bar{\nu}\right)$ and $\operatorname{Br}\left(K_{L} \rightarrow\right.$ $\left.\pi^{0} \nu \bar{\nu}\right)$ offer an interesting measurement of $\sin (2 \beta)$ without hadronic uncertainties. Choosing $\operatorname{Br}\left(K^{+} \rightarrow \pi^{+} \nu \bar{\nu}\right)=(1.0 \pm 0.1) \cdot 10^{-10}$ and $\operatorname{Br}\left(K_{L} \rightarrow \pi^{0} \nu \bar{\nu}\right)=(2.5 \pm 0.25) \cdot 10^{-11}$, 


\begin{tabular}{|c||c||c|c|c|}
\hline & Central & $I$ & $I I$ & $I I I$ \\
\hline$R_{t}$ & 1.00 & \pm 0.10 & \pm 0.05 & \pm 0.03 \\
\hline $\sin (2 \beta)$ & 0.70 & \pm 0.06 & \pm 0.02 & \pm 0.01 \\
\hline \hline$\varrho$ & 0.076 & \pm 0.111 & \pm 0.053 & \pm 0.031 \\
\hline$\eta$ & 0.388 & \pm 0.079 & \pm 0.033 & \pm 0.019 \\
\hline$\left|V_{u b} / V_{c b}\right|$ & 0.087 & \pm 0.014 & \pm 0.005 & \pm 0.003 \\
\hline
\end{tabular}

Table 5: Determinations of $\varrho$ and $\eta$ using $R_{t}$ and $\sin (2 \beta)$.

one finds 130

$$
\sin (2 \beta)=0.60 \pm 0.06 \pm 0.03 \pm 0.02
$$

where the first error is "experimental", the second represents the uncertainty in $m_{c}$ and $\Lambda_{\overline{M S}}$ and the last is due to the residual renormalization scale uncertainties. The $m_{t}$ and $V_{c b}$ dependences are negligible. This determination of $\sin (2 \beta)$ is competitive with the one expected at the B-factories at the beginning of the next decade.

Other future visions can be found in [37, 128, 129].

\section{Summary}

In this review we have discussed the most interesting quantities in B physics which when measured should have an important impact on our understanding of $\mathrm{CP}$ violation and quark mixing. We have also stressed that $\mathrm{K}$ physics can also offer an important contribution to this issue.

In this review we have concentrated on rare decays and $\mathrm{CP}$ violation in the standard model. The structure of rare decays and of $\mathrm{CP}$ violation in extensions of the standard model may deviate from this picture. Consequently the situation in this field could turn out to be very different from the one presented here. However in order to distinguish the standard model predictions from the predictions of its extensions it is essential that the theoretical calculations reach acceptable precision. In this context we have emphasized the importance of the QCD calculations in rare and $\mathrm{CP}$ violating decays. During the recent years a considerable progress has been made in this field through the computation of NLO contributions to a large class of decays [15]. This effort reduced considerably the theoretical uncertainties in the relevant formulae and thereby improved the determination of the CKM parameters to be achieved in future experiments. At the same time it should be stressed that whereas the theoretical status of QCD calculations for rare semileptonic decays like $K \rightarrow \pi \nu \bar{\nu}, B \rightarrow \mu \bar{\mu}, B \rightarrow X_{s} e^{+} e^{-}$is fully satisfactory and the status of $B \rightarrow X_{s} \gamma$ should improve in the coming years, a lot remains to be done in a large class of non-leptonic decays or transitions where non-perturbative uncertainties remain sizable. 
Clearly an exciting future is ahead of us. Let us just imagine that the following quantities have been measured to an acceptable accuracy:

- $\left|V_{u b} / V_{c b}\right|,\left|V_{c b}\right|, m_{t}$

- $\operatorname{Br}\left(K^{+} \rightarrow \pi^{+} \nu \bar{\nu}\right), \operatorname{Br}\left(K_{L} \rightarrow \pi^{0} \nu \bar{\nu}\right), \operatorname{Br}\left(B_{s} \rightarrow \mu^{+} \mu^{-}\right)$

- $B_{s}^{0}-\bar{B}_{s}^{0}$ - mixing

- $B \rightarrow X_{s} \gamma$ and $B \rightarrow X_{s} e^{+} e^{-}$

- $(\alpha, \beta, \gamma)$ in $\mathrm{CP}$ asymmetries in $\mathrm{B}$ decays.

- $\varepsilon^{\prime} / \varepsilon$ and $K_{L} \rightarrow \pi^{0} e^{+} e^{-}$.

and various non-perturbative parameters like $B_{K}, F_{B} \sqrt{B}$, and $\left(B_{6}, B_{8}\right)$ in $\varepsilon^{\prime} / \varepsilon$ have been calculated to an acceptable precision. With all these things at our disposal we could really get a great insight into the physics of CP violation and quark mixing and in particular find possible signals of some new physics beyond the standard model. At present all this is only a dream but already in ten years, at "Beauty 05", it could turn out to be reality!

\section{Final Remarks}

"Beauty 95" will remain in my memory for a long time and I am sure that this will also be the case for all participants. It was a splendid meeting run by the first lady Sue Geddes and the three musketeers Roger Cashmore, Neville Harnew and Peter Schlein helped by the team consisting of David Bailey, Lino Demaria, Anish Grewal, Chris Parkes, Armin Reichold and Davide Vite. They created five most enjoyable days in the scenery of Wadham College. Many thanks to them!

Yet nothing can be perfect. There was something missing at this meeting. I am referring here to four missing lambs at the Maytime Inn, which in the case of Fernando, Michael, Simone and myself, have been replaced by four dull chickens. Let us hope that during "Beauty 00" or "Beauty 05", held hopefully in Oxford again, the Maytime Inn will either find the missing lambs or serve us penguins instead.

\section{References}

[1] R.P. Feynman and M. Gell-Mann, Phys. Rev. 109 (1958) 193.

[2] A.J. Buras, M.Jamin and M.E. Lautenbacher, Nucl. Phys. B 408 (1993) 209.

[3] M. Neubert Physics Reports, 245 (1994) 259. 
[4] G. Buchalla, A.J. Buras and M.K. Harlander, Nucl. Phys. B 349 (1991) 1 .

[5] A.J. Buras, W. Slominski and H. Steger, Nucl. Phys. B 238 (1984) 529; Nucl. Phys. B 245 (1984) 269.

[6] T. Inami and C.S. Lim, Progr. Theor. Phys. 65 (1981) 297.

[7] J. Chay, H. Georgi and B. Grinstein, Phys. Lett. B247 (1990) 399.

[8] J. D. Bjorken, I. Dunietz and J. Taron, Nucl. Phys. B371 (1992) 111.

[9] I. I. Bigi, B. Blok, M. Shifman, N. G. Uraltsev and A. I. Vainshtein, in "B-Decays" (2nd Edition) edited by S. Stone, World Scientific (1994) page 132. I.I. Bigi et al, Phys. Lett. B 293 (1992) 430; Erratum 297 (1993) 477; Phys. Lett. B 323 (1994) 408; Phys. Rev. Lett. 71 (1993) 496;

[10] A.V. Manohar and M.B. Wise Phys. Rev. D 49 (1994) 1310; A.F. Falk, M.Luke, and M.J. Savage, Phys. Rev. D 49 (1994) 3367;

T. Mannel, Nucl. Phys. B413 (1994) 396; M. Neubert, Phys. Rev. D 49 (1994) 3392 and 4623 ;

[11] I.I. Bigi et al. Phys. Rev. D 50 (1994) 2234; M. Beneke, V.M. Braun and V.I. Zakharov, Phys. Rev. Lett. 73 (1994) 3058; M. Beneke and V.M. Braun, Nucl. Phys. B426 (1994) 301.

[12] P. Ball, M. Beneke and V.M. Braun, CERN-TH/95-65, hep-ph/9503492.

[13] G. Altarelli, G. Curci, G. Martinelli and S. Petrarca, Nucl. Phys. B 187 (1981) 461.

[14] A.J. Buras and P.H. Weisz, Nucl. Phys. B 333 (1990) 66.

[15] G. Buchalla, A.J. Buras and M. Lautenbacher, in preparation.

[16] A.J. Buras, M.Jamin, M.E. Lautenbacher and P.H. Weisz, Nucl. Phys. B 370 (1992) 69; Nucl. Phys. B 400 (1993) 37.

[17] A.J. Buras, M.Jamin and M.E. Lautenbacher, Nucl. Phys. B 400 (1993) 75 .

[18] M. Ciuchini, E. Franco, G. Martinelli and L. Reina, Phys. Lett. B 301 (1993) 263.

[19] M. Ciuchini, E. Franco, G. Martinelli and L. Reina, Nucl. Phys. B 415 (1994) 403.

[20] M.Misiak And M. Münz, Phys. Lett. B344 (1995) 308. 
[21] G. Buchalla, Nucl. Phys. B 391 (1993) 501.

[22] E. Bagan, P.Ball, V.M. Braun and P.Gosdzinsky, Nucl. Phys. B 432 (1994) 3; E. Bagan et al., Phys. Lett. B 342 (1995) 362; B 351 (1995) 546;

[23] M. Jamin And A. Pich, Nucl. Phys. B425 (1994) 15.

[24] S. Herrlich and U. Nierste, Nucl. Phys. B419 (1994) 292.

[25] A.J. Buras, M. Jamin, And P.H. Weisz, Nucl. Phys. B 347 (1990) 491.

[26] S. Herrlich and U. Nierste TUM-T31-81/95; hep-ph 9507262

[27] G. Buchalla And A.J. Buras, Nucl. Phys. B 398 (1993) 285.

[28] G. Buchalla and A.J. Buras, Nucl. Phys. B 400 (1993) 225.

[29] G. Buchalla And A.J. Buras, Nucl. Phys. B 412 (1994) 106.

[30] G. Buchalla And A.J. Buras, Phys. Lett. B 336 (1994) 263.

[31] A. J. Buras, M. E. Lautenbacher, M. Misiak and M. Münz, Nucl. Phys. B423 (1994) 349.

[32] M. Misiak, Nucl. Phys. B393 (1993) 23; Erratum, Nucl. Phys. B439 (1995) 461.

[33] A.J. Buras and M. Münz, Phys. Rev. D 52 (1995) 186.

[34] N. Cabibbo, Phys. Rev. Lett. 10 (1963) 531.

[35] M. Kobayashi and K. Maskawa, Prog. Theor. Phys. 49 (1973) 652.

[36] L. Wolfenstein, Phys. Rev. Lett. 51 (1983) 1945.

[37] A.J. Buras, M.E. Lautenbacher and G. Ostermaier, Phys. Rev. D 50 (1994) 3433.

[38] Particle Data Group, Phys. Rev. D 50 (1994) 1.

[39] F. Abe et al., CDF, Phys. Rev. D 50 (1994) 2966, Phys. Rev. Lett. 73 (1994) 225, Phys. Rev. D 51 (1995) 4623.

[40] S. ABACHI et al.,D0, FERMILAB-PUB-95/028E (1995).

[41] R. Patterson, in Proc. of the XXVII Int. Conf. on High Energy Physics, (Glasgow 94), edited by P.J. BUSSEY and I.G. KNOWLES (IOP Publications Ltd., Bristol,1995), Vol,p.149.

[42] M. Neubert, Phys. Lett. B 338 (1994) 84. 
[43] M. Shifman, N.G. Uraltsev and A. Vainshtein, Phys. Rev. D 51 (1995) 2217.

[44] N.G. Uraltsev, TPI-MINN-95/5-T.

[45] E. Thorndike (CLEO), EPS-HEP Conference, Brussels, July, 1995.

[46] A.J. Buras, Phys. Lett. B 317 (1993) 449.

[47] S. Sharpe, Nucl. Phys. (Proc. Suppl.) B34 (1994) 403;

[48] N. Ishizuka et al., Phys. Rev. Lett. 71 (1993) 24.

[49] W.A. Bardeen, A.J. Buras and J.-M. Gérard, Phys. Lett. B211 (1988) 343; J-M. GÉrard, Acta Physica Polonica B21 (1990) 257.

[50] J. Bijnens and J. Prades, Nucl. Phys. B 444 (1995) 523.

[51] A. Pich and E. de Rafael, Phys. Lett. B158 (1985) 477; J. Prades et al, Z. Phys. C51 (1991) 287.

[52] J.F. Donoghue, E. Golowich and B.R. Holstein, Phys. Lett. B119 (1982) 412 .

[53] C.T. Sachrajda, in "B-Decays" (2nd Edition) edited by S. Stone, World Scientific (1994) page 602.

[54] A. Duncan, E. Eichten, J. Flynn, B. Hill, G. Hockney and H. Thacker, Phys. Rev. D 51 (1995) 5101.

[55] C.W. Bernard, J.N. Labrenz and A. Soni, Phys. Rev. D 49 (1994) 2536;

T. Draper and C. McNeile, Nucl. Phys. (Proc. Suppl.) 34 (1994) 453.

[56] E. Bagan, P. Ball, V.M. Braun and H.G. Dosch, Phys. Lett. B 278 (1992) 457; M. Neubert, Phys. Rev. D 45 (1992) 2451;

[57] C.G. Boyd, B. Grinstein and R.F. Lebed, UCSD/PTH 94-27.

[58] S. NARISOn, Phys. Lett. B322 (1994) 247;

[59] G. Rizzo, these proceedings.

[60] A. Stocchi, these proceedings.

[61] R. Aleksan, Plenary talk, EPS-HeP Conference, Brussels, July, 1995.

[62] M. Ciuchini et AL. CERN-TH-7514-94, hep-ph/9501265 
[63] A. Ali and D. London, DESY 95-148, hep-ph 9508272 (1995).

[64] R.D. Peccei and K. Wang, Phys. Lett. B 349 (1995) 220.

[65] G. Altarelli and S. Petrarca, Phys. Lett. B 261 (1991) 303.

[66] T.E. Browder and K. Honscheid, UH-511-816-95, hep-ph/9501287, To Be published in Progress in Particle and Nuclear Physics 35.

[67] Q. Hokim And X.Y. Pham, Phys. Lett. B 122 (1983) 297; Ann. Phys. B 155 (1984) 202.

[68] M.B. Voloshin, Phys. Rev. D 51 (1995) 3948;

[69] I.I. Bigi et Al., Phys. Lett. B 323 (1994) 408; A.F. FAlk, M.B. Wise And I. Dunietz, Phys. Rev. D 51 (1995) 1183; I. Dunietz, (HEP-PH/9501287).

[70] G. Buchalla, I. Dunietz and H. Yamamoto, FERMilAB-PUB-95/167$\mathrm{T}$.

[71] R. RÜCKL, THESE PROCEEDINGS.

[72] M. Wirbel, B. Stech and M. Bauer, Z.Phys. C29 (1985) 637.

[73] A.J. Buras, Nucl. Phys. B 434 (1995) 606.

[74] J.D. BJorken, Nucl. Phys. (Proc. Suppl.) B 434 (1989) 325; SLAC-PUB5389.

[75] S. Bertolini, F. Borzumati and A. Masiero, Phys. Rev. Lett. 59 (1987) 180.

[76] N. G. Deshpande, P. Lo, J. Trampetic, G. Eilam and P. Singer Phys. Rev. Lett. 59 (1987) 183.

[77] B. Grinstein, R. Springer and M.B. Wise, Nucl. Phys. B339 (1990) 269.

[78] R. Grigjanis, P.J. O'Donnell, M. Sutherland and H. Navelet, Phys. Lett. B213 (1988) 355; Phys. Lett. B286 (1992) 413 E.

[79] M. Ciuchini, E. Franco, G. Martinelli, L. Reina and L. Silvestrini, Phys. Lett. B316 (1993) 127.

[80] M. Ciuchini, E. Franco, L. Reina and L. Silvestrini, Nucl. Phys. B421 (1994) 41.

[81] G. Cella, G. Curci, G. Ricciardi and A. Viceré, Phys. Lett. B325 (1994) 227. 
[82] G. Cella, G. Curci, G. Ricciardi and A. Viceré, Nucl. Phys. B431 (1994) 417.

[83] K. Adel and Y.P. Yao, Modern Physics Letters A8 (1993) 1679; Phys. Rev. D 49 (1994) 4945.

[84] A. Ali, and C. Greub, Z.Phys. C60 (1993) 433.

[85] A. Ali, and C. Greub, Z.Phys. C49 (1991) 431, Phys. Lett. B259 (1991) 182.

[86] A. J. Buras, M. Misiak, M. Münz and S. Pokorski, Nucl. Phys. B424 (1994) 374 .

[87] R. Ammar et. al. (CLEO), Phys. Rev. Lett. 71 (1993) 674.

[88] M.S. Alam et. al (CLEO), Phys. Rev. Lett. 74 (1995) 2885.

[89] M. Ciuchini, E. Franco, G.Martinelli, L. Reina and L. Silvestrini, Phys. Lett. B334 (1994) 137.

[90] M. Münz, PhD Thesis, Technical University Munich (1995).

[91] A. ALI, DESY 95-157, hep-ph/9508335

[92] W. S. Hou, R. I. Willey and A. Soni, Phys. Rev. Lett. 58 (1987) 1608.

[93] B. Grinstein, M. J. Savage and M. B. Wise, Nucl. Phys. B319 (1989) 271.

[94] R. Grigjanis, P. J. O’Donnell, M. Sutherland and H. Navelet, Phys. Lett. B223 (1989) 239.

[95] G. Cella, G. Ricciardi And A. Viceré, Phys. Lett. B258 (1991) 212.

[96] A. Falk, M. Luke and M.J. Savage, Phys. Rev. D 49 (1994) 3367.

[97] A. Ali, G.F. Giudice and T. Mannel, CERn-TH 7346/94, HePPH/9408213; C. Greub, A. Ioannissian and D. Wyler, Phys. Lett. B346 (1995) 149; G. Burdman, Fermilab-Pub-95/113-T, HeP-PH/9505352.

[98] M. Gronau, these Proceedings.

[99] Y. Nir and H.R. Quinn in " B Decays ", ed S. Stone (World Scientific, 1994), P. 520; I. Dunietz, IBID P.550 AND REFS. THEREIN.

[100] M. Gronau, Phys. Rev. Lett. 63 (1989) 1451, Phys. Lett. B 233 (1989) 479; I. Dunietz and J.L Rosner,Phys. Rev. D 34 (1986) 1404; R. Aleksan, I. Dunietz, B. Kayser and F. Le Diberder, Nucl. Phys. B 361 (1991) 141. 
[101] M. Gronau and D. Wyler, Phys. Lett. B 265 (1991) 172.

[102] M. Gronau and D. London, Phys. Rev. Lett. 65 (1990) 3381, Phys. Lett. B 253 (1991) 483; Y. Nir And H. Quinn, Phys. Rev. D 42 (1990) 1473, Phys. Rev. Lett. 67 (1991) 541;

[103] M. Gronau, J.L. Rosner and D. London, Phys. Rev. Lett. 73 (1994) 21.

[104] M. Gronau, O.F. Hernández, D. London and J.L. Rosner, Phys. Rev. D50 (1994) 4529.

[105] O.F. Hernández, D. London, M. Gronau and J.L. Rosner, Phys. Lett. B333 (1994) 500.

[106] I.I.Y. Bigi and A.I. Sanda, Nucl. Phys. B 193 (1981) 85.

[107] A.J. Buras and R. Fleischer, TUM-T31-96/95, hep-ph/9507460 (1995).

[108] G. Kramer and W.F. Palmer, DESY 95-131, hep-ph/9507329 (1995).

[109] N.G. Deshpande And X.-G. He, Phys. Rev. Lett. 74 (1995) 26.

[110] M. Gronau, O.F. Hernández, D. London and J.L. Rosner, TECHNION-PH-95-11, hep-ph/9504327 (1995).

[111] A.J. Buras and R. Fleischer, TUM-T31-95/95, hep-ph/9507303 (1995).

[112] R. Fleischer, TTP95-32, hep-ph/9509204. (1995).

[113] I. Dunietz, Phys. Lett. B270 (1991) 75.

[114] R. Aleksan, I. Dunietz and B. Kayser, Z.Phys. C54 (1992) 653.

[115] I. Dunietz, Snowmass B-Physics 1993, P.83.

[116] A.J. Buras and R. Fleischer, Phys. Lett. B341 (1995) 379.

[117] M. Gronau, O.F. Hernández, D. London and J.L. Rosner, TECHNION-PH-95-10, hep-ph/9504326 (1995).

[118] R. Fleischer, Z.Phys. C62 (1994) 81.

[119] R. Fleischer, Phys. Lett. B321 (1994) 259.

[120] R. Fleischer, Phys. Lett. B332 (1994) 419.

[121] N.G. Deshrpande and X.-G. He,, Phys. Lett. B 336 (1994) 471. 
[122] N.G. Deshpande, X.-G. He and J. Trampetic, Phys. Lett. B345 (1995) 547.

[123] D. Du And M. YANG, BIHEP-TH-95-8, hep-ph/9503278 (1995).

[124] N.G. Deshpande and X.-G. He, OITS-576, hep-ph/9505369 (1995).

[125] R. Fleischer, Phys. Lett. B 341 (1994) 205.

[126] L. Wolfenstein, Phys. Rev. Lett. 13 (1964) 562.

[127] B. Winstein, Phys. Rev. Lett. 68 (1992) 1271.

[128] A.J. Buras, Phys. Lett. B 333 (1994) 476.

[129] A.J. Buras, Acta Physica Polonica, 26 (1995) 755.

[130] G. Buchalla and A.J. Buras, Phys. Lett. B 333 (1994) 221. 
This figure "fig1-1.png" is available in "png" format from: http://arxiv.org/ps/hep-ph/9509329v2 
This figure "fig2-1.png" is available in "png" format from: http://arxiv.org/ps/hep-ph/9509329v2 
This figure "fig1-2.png" is available in "png" format from: http://arxiv.org/ps/hep-ph/9509329v2 
This figure "fig2-2.png" is available in "png" format from: http://arxiv.org/ps/hep-ph/9509329v2 
This figure "fig1-3.png" is available in "png" format from: http://arxiv.org/ps/hep-ph/9509329v2 